\title{
The Dilution Dependency of Multigroup Uncertainties
}

\author{
M. R. Ball, ${ }^{1,2}$ C. McEwan, ${ }^{1}$ D. R. Novog, ${ }^{1}$ and J. C. Luxat ${ }^{1}$ \\ ${ }^{1}$ McMaster University, 1280 Main Street West, Hamilton, ON, Canada L8S 4L8 \\ ${ }^{2}$ Department of Engineering Physics, McMaster University, Room JHE-A315, 1280 Main Street West, Hamilton, ON, Canada L8S 4L8
}

Correspondence should be addressed to M. R. Ball; themattball@gmail.com

Received 4 February 2014; Revised 26 June 2014; Accepted 29 July 2014; Published 2 December 2014

Academic Editor: Keith E. Holbert

Copyright (C) 2014 M. R. Ball et al. This is an open access article distributed under the Creative Commons Attribution License, which permits unrestricted use, distribution, and reproduction in any medium, provided the original work is properly cited.

\begin{abstract}
The propagation of nuclear data uncertainties through reactor physics calculation has received attention through the Organization for Economic Cooperation and Development-Nuclear Energy Agency's Uncertainty Analysis in Modelling (UAM) benchmark. A common strategy for performing lattice physics uncertainty analysis involves starting with nuclear data and covariance matrix which is typically available at infinite dilution. To describe the uncertainty of all multigroup physics parameters-including those at finite dilution-additional calculations must be performed that relate uncertainties in an infinite dilution cross-section to those at the problem dilution. Two potential methods for propagating dilution-related uncertainties were studied in this work. The first assumed a correlation between continuous-energy and multigroup cross-sectional data and uncertainties, which is convenient for direct implementation in lattice physics codes. The second is based on a more rigorous approach involving the Monte Carlo sampling of resonance parameters in evaluated nuclear data using the TALYS software. When applied to a light water fuel cell, the two approaches show significant differences, indicating that the assumption of the first method did not capture the complexity of physics parameter data uncertainties. It was found that the covariance of problem-dilution multigroup parameters for selected neutron cross-sections can vary significantly from their infinite-dilution counterparts.
\end{abstract}

\section{Introduction}

Uncertainty analysis involves statistically quantifying the range of variation of uncertain output responses that are attributable to the variation of uncertain input parameters or models. Often in operational and safety analysis of nuclear reactors some level of uncertainty is attributed to the prediction of key variables and often these uncertainties are directly factored into operating margins and limits. The first exercises of the uncertainty analysis in modeling (UAM) benchmark [1] focus primarily on the uncertainty attributable to microscopic multigroup neutron interaction cross-sections and their propagation through lattice physics transport calculations and in subsequent exercises ultimately trough full-core diffusion predictions. The range of variation of those uncertain input parameters is characterized by variance-covariance matrices.

In literature, there are a variety of terms that are used to classify the mechanisms through which nuclear data uncertainties propagate through reactor lattice calculations, such as explicit, implicit, direct and indirect, broad-structure, fine-structure, and others [2-5]. For the purposes of this paper there are four high-level mechanisms by which uncertainty in microscopic cross-section will be propagated to the solutions of lattice physics calculations $\left(k_{\infty}\right.$ and homogenized few-group properties). Firstly, the effects from crosssection uncertainties which directly perturb the loss and gain operators of the Bolztmann transport equation are called the explicit mechanism (or explicit effect). The second through fourth effects are denoted as implicit mechanisms, in that the perturbations come through the solution and methods used to solve the transport equation. The second, termed the indirect flux mechanism, is attributed to changes in $k_{\infty}$ which come indirectly through perturbations in the neutron flux spectra resulting from cross-section perturbations. Such effects are present in both continuous energy and multigroup solutions of the transport equation. The third and fourth mechanisms arise from the treatment of energy in the transport equation in discretized energy form and are elsewhere classified as spectral fine-structure effects [2-4], 
as they involve the perturbation of the neutron flux on a scale much smaller than energy group widths. One of the first available tools for uncertainty analysis with a rigorous treatment of sensitivities that include implicit effects was developed by Oak Ridge National Laboratory (ORNL) and implemented in the code TSUNAMI [5-7].

The background cross-section mechanism is the third mechanism and arises because a perturbation of a multigroup nuclear data cross-section will perturb the background cross-section, $\sigma_{0}$, seen by all other admixed nuclides at each interval in the energy discretization. Therefore crosssection uncertainties propagate through the self-shielding and affect the uncertainty in reactions for other isotopes. For example, in LWR fuel, a perturbation of $\sigma_{\gamma}^{\mathrm{U} 238}$ may have a greater influence on $k_{\infty}$ through its perturbation of the background cross-section used for self-shielding of ${ }^{235} \mathrm{U}$ than it will directly by perturbing transport equation operators on its own. The fourth mechanism is an inherent dilutiondependency mechanism related to cross-section uncertainty that emerges because a perturbation is propagated through the weighting function (flux estimate) for energy domain discretization. Therefore, combining these latter two mechanisms for a given perturbation of the underlying nuclear cross-sections, the variation of its corresponding multigroup cross-section will depend on the background cross-section attributed to admixed nuclides as well as the flux used in the weighting process. Quite often in analyses, the above effects are integral in the calculations and are not broken down into its component mechanisms. Further discussion on the background and dilution effects is provided in Section 2.

All uncertainty analysis methodologies must utilize a given reference nuclear data set, sometimes in continuous energy or perhaps already processed into multigroup crosssections, and an associated variance-covariance uncertainty matrix (hereafter referred to as covariance data). A commonly used set of covariance data is 44GROUPCOV [8], evaluated by ORNL, which is a 44-energy group covariance library describing the uncertainties for a large number of isotopes. The ORNL covariance data are currently not indexed to either temperature or dilution-two parameters which are generally necessary in reference case computations. Therefore quite often an assumption must be made in applying this covariance information at problem specific temperature and dilution levels. Covariance can also be calculated by the ERRORR module of the cross-section processing code NJOY [9] based on the information contained in the continuous energy nuclear data libraries. However, ERRORR covariance data are similarly restricted to infinite-dilution data. The common strategy for lattice physics uncertainty propagation is to use the infinite-dilution covariance dataset for all multigroup cross-sections present in the lattice. When deemed necessary, some procedures correct for implicit mechanisms by including the self-shielding calculation in the uncertainty propagation sequence [10]. Other methods, recognizing that the implicit effect can be small for many lattice types, neglect it and apply the infinite-dilution covariance to problemdependent (resonance self-shielded) cross-sections directly. The subtlety here is that while many codes adequately capture the role of dilution on the reference cross-sections at problem specific conditions (i.e., through self-shielding calculations) the effect of dilution on the transformation between continuous and multigroup uncertainties is more complex and affects subsequent uncertainties of both multigroup and few-group parameters. The focus of this paper is to examine the effect of dilution dependence of the covariance libraries applied in lattice physics calculations and to study its effect on neutron multiplication and few-group cross-sections.

Since the process of uncertainty propagation through resonance self-shielding is highly tied to background crosssection and this background is used in the assessment of the unperturbed reference nuclear data it is worthwhile to highlight some of the important features in standard nuclear data processing. In the reference case calculations, continuous energy cross-sections from a nuclear data library are typically processed with a code like NJOY to obtain multigroup nuclear data. Since no problem specific dilution data are applied at this time, the processing is performed for a parametric range of dilutions. Hence the multigroup nuclear data output at this stage consists of a table of reference multigroup cross-sections indexed to background crosssection. Downstream nuclear transport codes then utilize problem specific geometry and dilution and interpolate the required background cross-section to be applied during the resonance self-shielding stage. At this point the lattice physics code may then solve the neutron transport equation and determine the local flux distribution and reaction rates, as well as all subsequent processing to obtain few-group crosssections.

The ERRORR module of NJOY processes the uncertainty information contained in a fundamental nuclear data library and collapses it in the energy domain to a multigroup structure that characterizes the uncertainty of multigroup neutron cross-sections at infinite dilution. It should be noted at this stage that at infinite dilution the neutron flux remains constant when cross-sections are perturbed and hence at infinite dilution no special treatment is required. However, at finite dilutions, variation of the cross-section in the neighbourhood of a resonance peak will lead to changes in the shape of the corresponding neutron flux depression, as illustrated in Figure 1. The resonance self-shielding effect that arises from flux depressions causes the multigroup cross-section to be perturbed as a result of two contributions: the first is from the perturbation in the cross-section that is being averaged and the second is from the perturbation of the flux which acts as the weighting function for the averaging [11]. Therefore the variation of the resulting self-shielded multigroup crosssection will be dilution-dependent since it depends in part on the perturbation of the neutron flux, and the perturbation of the neutron flux depends on the dilution of the nuclide to which the cross-section belongs; the lower the dilution of the nuclide, the larger the neutron flux perturbation. As a result the uncertainty transformation from fundamental nuclear data through to multigroup libraries and eventually to problem specific applications must allow for the role of dilution in the propagation sequence. Fundamentally this can be summarized in the following statement. 


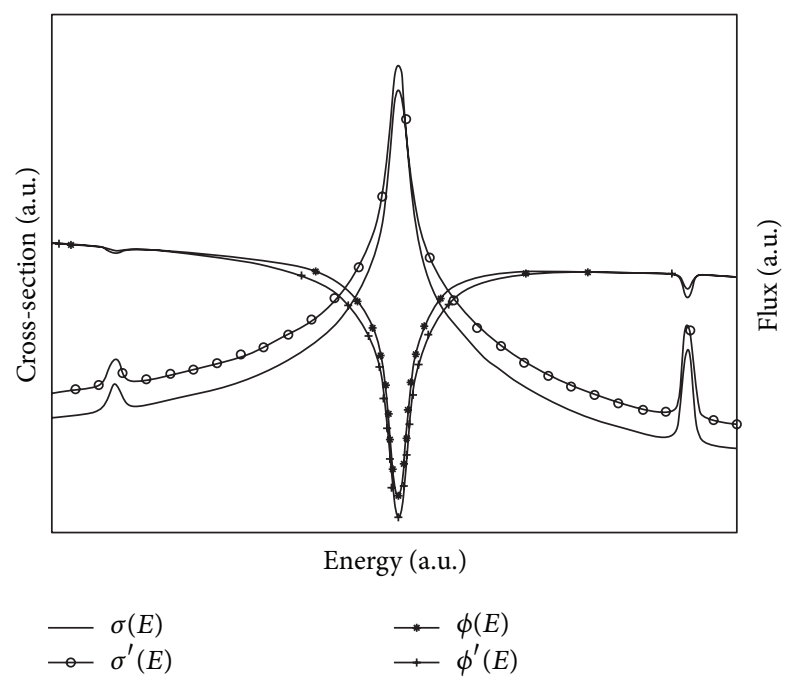

Figure 1: Cross-section, $\sigma(E)$, and flux, $\phi(E)$, with their perturbed (marked with prime) counterparts.

Since any multigroup data, in, for example, 44 or 69 energy groups, must be indexed to dilution in some way, then it may also be necessary for the treatment of covariance data to be indexed in a similar manner.

\section{Multigroup Formulation and Sensitivities}

Multigroup cross-sections are computed through the averaging of continuous energy cross-sections weighted by the neutron flux over each interval of energy. An estimate of a problem-independent flux within each energy group is therefore necessary and is calculated by cross-section processing codes such as NJOY. In the case of NJOY specifically, the flux at intermediate neutron energies (from a few $\mathrm{eV}$ to several $\mathrm{keV}$ ), where resonance self-shielding is important, is estimated either by the Bondarenko narrow resonance model [12] or by the NJOY flux calculator. For simplicity, this paper considers flux estimation by the Bondarenko method exclusively, but a similar procedure could be considered for the flux calculator. In the remainder of this section, the Bondarenko narrow resonance model is described in brief, when considering both nominally valued and perturbed cross-sections.

The first approximation of the Bondarenko model is that the continuously varying neutron flux takes on the form seen in (1) at slowing-down energies:

$$
\phi(E) \propto \frac{1}{E}\left(\frac{1}{\Sigma_{t}(E)}\right) .
$$

In (1), $\Sigma_{t}(E)$ is the total macroscopic cross-section in the flux medium at energy $E$. The total macroscopic cross-section can be expanded into its constituent microscopic cross-sections, as in (2), where $N^{j}$ is the atom density of nuclide $j$ and nuclide $i$ is an absorber whose cross-sections are to be selfshielded:

$$
\begin{aligned}
\Sigma_{t} & =N^{i} \sigma_{t}^{i}+\sum_{j \neq i} N^{j} \sigma_{t}^{j}=N^{i}\left(\sigma_{t}^{i}+\sum_{j \neq i} \frac{N^{j}}{N^{i}} \sigma_{t}^{j}\right) \\
& =N^{i}\left(\sigma_{t}^{i}+\sigma_{0}\right) .
\end{aligned}
$$

A new parameter, $\sigma_{0}$, has been introduced in (2), referred to as the background cross-section. The background cross-section is the total macroscopic cross-section of all other admixed isotopes in per-absorber atom units and is assumed constant within the energy group interval, $g$.

Neutron flux is used as the weighting function in the energy discretization process, leading to the formulation of a multigroup parameter, $\sigma_{x, g}^{i}\left(\sigma_{0}\right)$, of reaction type $x$, belonging to an absorbing nuclide $i$ in energy group, $g$, as shown in the following:

$$
\sigma_{x, g}^{i}\left(\sigma_{0}\right)=\frac{\int_{g}\left(\sigma_{x}^{i}(\mu) /\left(\sigma_{t}^{i}(\mu)+\sigma_{0}\right)\right) d \mu}{\int_{g}\left(d \mu /\left(\sigma_{t}^{i}(\mu)+\sigma_{0}\right)\right)} .
$$

Note that in (3) continuously varying parameters are expressed as a function of neutron lethargy, $\mu$, rather than energy. Also, the macroscopic cross-section $\Sigma_{t}$ has been replaced by the expression in (2). It is clear from (3) that the multigroup parameter is a function of the parameter $\sigma_{0}$, and when $\sigma_{0}$ approaches infinity, (3) reduces to (4). Consider

$$
\sigma_{x, g}^{i, \infty}=\frac{\int_{g} \sigma_{x}^{i}(\mu) d \mu}{\int_{g} d \mu}
$$

By the nature of the resonance self-shielding effect, $\sigma_{x, g}^{i}\left(\sigma_{0}\right)$ is less than $\sigma_{x, g}^{i, \infty}$ for all finite values of $\sigma_{0}$, as the flux depression leads to a decrease in the reaction rate. The reduction is commonly expressed using self-shielding factors, $f$ [13]. For a particular cross-section, self-shielding factors are a function of energy group and background cross-section and are defined as

$$
f_{x, g}^{i}\left(\sigma_{0}\right)=\frac{\sigma_{x, g}^{i}\left(\sigma_{0}\right)}{\sigma_{x, g}^{i, \infty}}
$$

The application of these treatments for reference crosssection processing is widely accepted and forms the basis for determining the multigroup cross-sections required by many lattice physics codes $[9,14]$. The extension of these treatments for development of relevant covariance matrices as a function of dilution is discussed below.

Given the Bondarenko formalism, the background crosssection mechanism and dilution-dependency mechanisms can be conceptually distinguished by the following example. Suppose a material is consisting of a mixture of two isotopes, $i$ and $j$. Further suppose the cross-sections of isotope $i$ are uncertain, while the cross-sections of isotope $j$ are precisely known. In this example, the multigroup constants for both 
isotopes $i$ and $j$ will be uncertain but each by a different mechanism. The group constants, $\sigma_{x, g}^{i}\left(\sigma_{0}\right)$, of isotope $i$, will be uncertain via the uncertainty in $\sigma_{x}^{i}(\mu)$ and $\sigma_{t}^{i}(\mu)$ propagating through (3). The background cross-section, $\sigma_{0}$, seen by isotope $i$, is constant and does not vary except according to atom densities of isotopes $i$ and $j$ in the mixture. The uncertainty of the group constants of isotope $i$ is therefore dilution-dependent. Conversely, the group constants, $\sigma_{x, g}^{j}\left(\sigma_{0}\right)$, of isotope $j$ will be uncertain solely because the background cross-section seen by isotope $j$ will be uncertain and will propagate through (3), since the background cross-section of isotope $j$ is a function of $\sigma_{t}^{i}(\mu)$. Isotope $j$ group constants are therefore uncertain as a result of the background crosssection mechanism; its background cross-section is uncertain even when the atom densities of the two isotopes are specified exactly. Therefore it can be seen that although isotope $j$ data had no uncertainty in continuous energy, after propagation through energy discretization and self-shielding it will have some uncertainty arising from the uncertainty in the background cross-section. It should also be noted that all resonance self-shielding effects can potentially become less important as energy group widths become narrower and therefore are sensitive to particular energy group structures.

\section{Dilution-Dependence of Uncertainty according to a Full Correlation Assumption}

To apply infinite-dilution covariance directly to problemdilution multigroup cross-sections, sensitivity must be calculated that relates the variation of the former to the variation of the latter. Strictly, the cross-section curve in continuous energy is necessary to compute such sensitivity exactly, but often only discretized multigroup information is available during a lattice calculation (for example, in many industrial applications there exists a predefined and standardized multigroup library which has been vetted and adjusted against experimental data for specific reactor designs; therefore such data are usually not recomputed from continuous nuclear data on a regular basis). Without continuous data, the sensitivity can be approximated from multigroup data by adopting an assumption regarding the nature of the crosssection perturbation within a given energy group, which will be referred to in this paper as the "full correlation within-group" (FCWG) assumption. By assuming that the points along the continuous cross-section are fully correlated within each energy group, thereby assuming that the shape of the cross-section spectra is fixed within an energy range, and with uncertainty only in its magnitude, the variation of the infinite-dilution cross-section can be related to that at a problem dilution, by propagating a perturbation in continuous energy through the Bondarenko formalism as shown below.

Consider a continuous energy cross-section, $\sigma_{x}^{i}(\mu)$, subjected to a perturbation, $\left(1+\varepsilon_{x}^{i}\right)$, resulting in a perturbed cross-section, $\sigma_{x}^{\prime i}(\mu)$, as shown in the following:

$$
\sigma_{x}^{\prime i}(\mu)=\left(1+\varepsilon_{x}^{i}\right) \sigma_{x}^{i}(\mu)
$$

According to the aforementioned FCWG assumption, $\varepsilon_{x}^{i}$ is constant within each discretized energy group. By substituting (6) into (4), perturbed infinitely dilute multigroup crosssection for some energy group, $g$, can be found:

$$
\begin{aligned}
\sigma_{x, \mathfrak{g}}^{\prime i, \infty} & =\frac{\int_{g} \sigma_{x}^{\prime i}(\mu) d \mu}{\int_{g} d \mu}=\frac{\int_{g}\left(1+\varepsilon_{x}^{i}\right) \sigma_{x}^{i}(\mu)}{\int_{g} d \mu} \\
& =\left(1+\varepsilon_{x}^{i}\right) \sigma_{x, \mathfrak{g}}^{i, \infty} .
\end{aligned}
$$

As seen in (7), a uniform perturbation of $\sigma(\mu)$ within an energy group results in infinite-dilution multigroup parameters that are perturbed by the same multiplier. However, at other dilutions the relationship between continuous energy perturbations and the perturbations seen in multigroup parameters is more complex:

$$
\begin{aligned}
& \sigma_{x, g}^{\prime i}\left(\sigma_{0}\right)=\frac{\int_{g}\left(\sigma_{x}^{\prime i}(\mu) /\left(\sigma_{t}^{\prime i}(\mu)+\sigma_{0}\right)\right) d \mu}{\int_{g}\left(d \mu /\left(\sigma_{t}^{\prime i}(\mu)+\sigma_{0}\right)\right)} \\
&=\left(1+\varepsilon_{x}^{i}\right) \frac{\int_{g}\left(\sigma_{x}^{i}(\mu) /\left(\sigma_{t}^{i}(\mu)+\sigma_{0} /\left(1+\varepsilon_{t}^{i}\right)\right)\right) d \mu}{\int_{g}\left(1 /\left(\sigma_{t}^{i}(\mu)+\sigma_{0} /\left(1+\varepsilon_{t}^{i}\right)\right)\right) d \mu} \\
&=\left(1+\varepsilon_{x}^{i}\right) \sigma_{x, g}^{i}\left(\sigma_{0}^{\prime}\right) \\
& \sigma_{0}^{\prime}=\frac{\sigma_{0}}{\left(1+\varepsilon_{t}^{i}\right)}
\end{aligned}
$$

As shown in (8) and (9), when a uniform perturbation is applied to a continuous cross-section within an energy group, $g$, the effect of that perturbation on the corresponding multigroup parameter can be determined from the unperturbed multigroup parameter evaluated at a perturbed background cross-section, $\sigma_{0}^{\prime}$.

As mentioned previously, often in industrial applications a prescribed reference set of multigroup nuclear data exists and these data have undergone rigorous evaluation, adjustments, and validation. Hence frequent reconstruction of such data from fundamental continuous nuclear data may not be desirable. When preexisting nuclear data are available only in multigroup form, it is not possible to fully reconstruct the covariance information that exists in the continuous spectra. In this situation (8) and (9) provide a method for approximating the difference in variation of parameters at various values of background cross-section. The variation of a multigroup cross-section at a problem dilution relative to infinite dilution can be used to quantify the uncertainty of the problem-dilution parameter in conjunction with an infinitedilution covariance matrix.

In the remainder of this section, the relative variation of the ${ }^{238} \mathrm{U}$ and ${ }^{235} \mathrm{U}$ radiative-capture cross-sections was computed at various values of background cross-section using the FCWG assumption. The cross-section processing code NJOY was first used to generate point-wise cross-sections on a fine energy grid that closely approximate (an NJOY RECONR tolerance parameter of $1 \mathrm{E}-5$ ) continuous data. To calculate 
TABLE 1: WIMS 69-group library resonance group boundaries.

\begin{tabular}{lc}
\hline Group & Upper energy $(\mathrm{eV})$ \\
\hline 15 & $9.12 \times 10^{3}$ \\
16 & $5.53 \times 10^{3}$ \\
17 & $3.52 \times 10^{3}$ \\
18 & $2.24 \times 10^{3}$ \\
19 & $1.43 \times 10^{3}$ \\
20 & $9.07 \times 10^{2}$ \\
21 & $3.67 \times 10^{2}$ \\
22 & $1.49 \times 10^{2}$ \\
23 & $7.55 \times 10^{1}$ \\
24 & $4.81 \times 10^{1}$ \\
25 & $2.77 \times 10^{1}$ \\
26 & $1.60 \times 10^{1}$ \\
27 & $9.88 \times 10^{0}$ \\
& $4.00 \times 10^{0}$ \\
\hline
\end{tabular}

relative variation, the point-wise data at all energies were perturbed by a small uniform multiplicative scaling, and a numerical solution of the Bondarenko self-shielding model produced the resulting perturbed multigroup data. The final multigroup collapsed cross-sections were determined based on energy domain discretization consistent with the 69-group WIMS energy structure [14]. Open-source NJOY input decks for this energy group structure are available through the WLUP project [15]. As ${ }^{238} \mathrm{U}$ is both a nuclide of particularly low dilution in typical LWR reactor systems and an important uncertainty contributor to LWR lattice $k_{\infty}$ uncertainty, it is especially sensitive to the change in variation at problem dilution compared to infinite dilution. Therefore to illustrate the concepts relevant in this paper the results will focus on the dilution-dependency of the covariance information centered on this isotope.

The 69-group energy structure includes 13 intermediate energy groups (groups 15 to 27) for which self-shielding parameters are included. It is those 13 groups where selfshielding is important and their upper and lower energy boundaries are listed in Table 1.

Figure 2 shows the relative uncertainty of resonance group $\sigma_{\gamma}^{\mathrm{U} 238}$ cross-section as a function of background crosssection, expressed as a fraction of the uncertainty at infinite dilution when assuming that the data are FCWG. Note that there are thirteen curves, one for each resonance energy group. For example, at $100 b$ of background cross-section, the uncertainty of $\sigma_{\gamma}^{\mathrm{U} 238}$ is less than $90 \%$ of that at infinite dilution for twelve of the thirteen resonance groups. The symbol " $\Delta$ " is used to denote standard deviation rather than " $\sigma$ " to avoid confusion with microscopic cross-sections. Figure 3 shows a similar set of curves for $\sigma_{\gamma}^{\mathrm{U} 235}$.

For most of the resonance groups, the group-wise crosssection uncertainty is very sensitive to background crosssection, especially when the background cross-sections are small (less than 1000b). Equivalently, the group-wise crosssections themselves are particularly insensitive to variations in the point-wise cross-sections at low dilutions; large

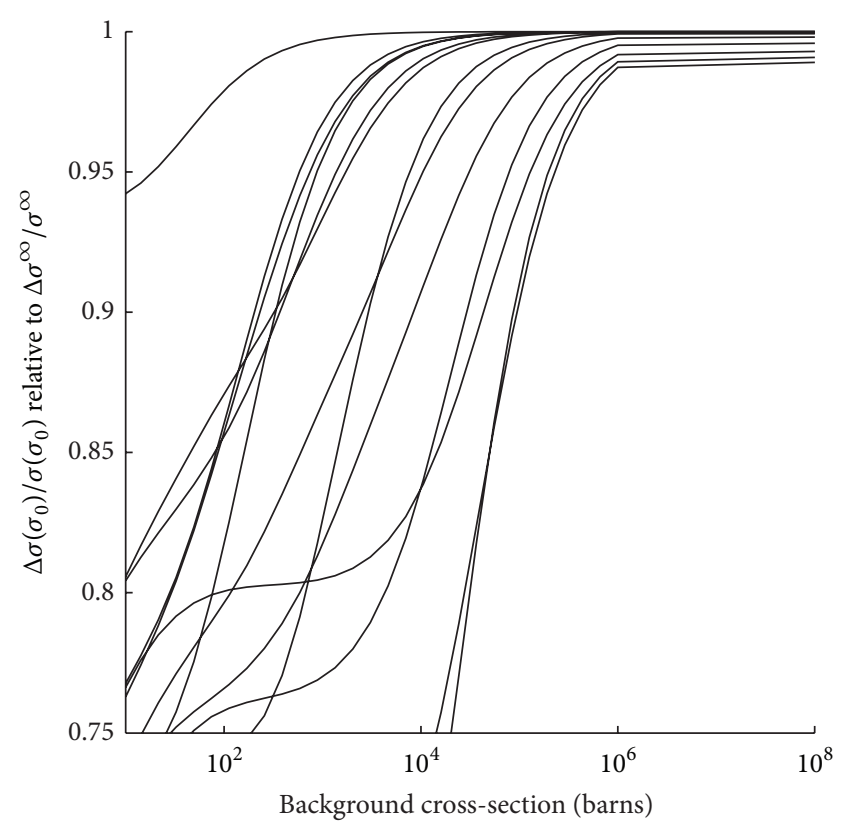

FIGURE 2: Resonance group $\Delta \sigma / \sigma$ versus dilution relative to infinite dilution of $\sigma_{\gamma}^{\mathrm{U} 238}$ using FCWG.

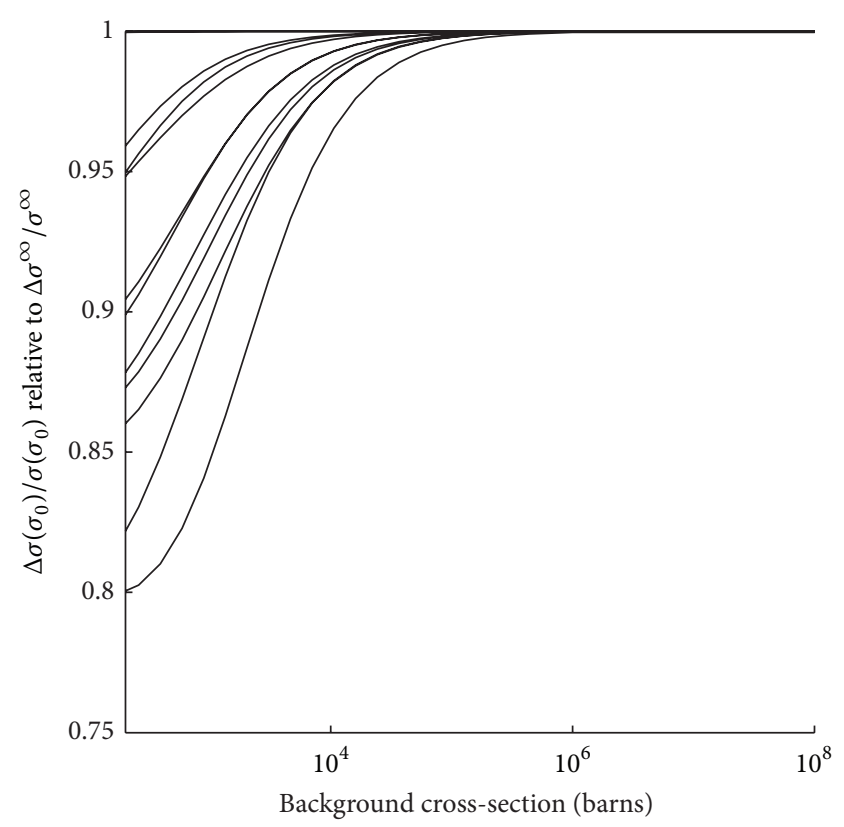

FIgURE 3: Resonance group $\Delta \sigma / \sigma$ versus dilution relative to infinite dilution of $\sigma_{\gamma}^{\mathrm{U} 235}$ using FCWG.

variations, or uncertainties, in the point-wise cross-sections result in much smaller variations in their group-wise counterparts. The value of the FCWG approximation is that it can be used to approximate covariance as a function of dilution whereas in many propagation methods dilution effects on the reference cross-section were captured but the underlying covariance was treated at infinite dilution. For situations that occur in industry where reference multigroup cross-section libraries exist and where it may be undesirable 


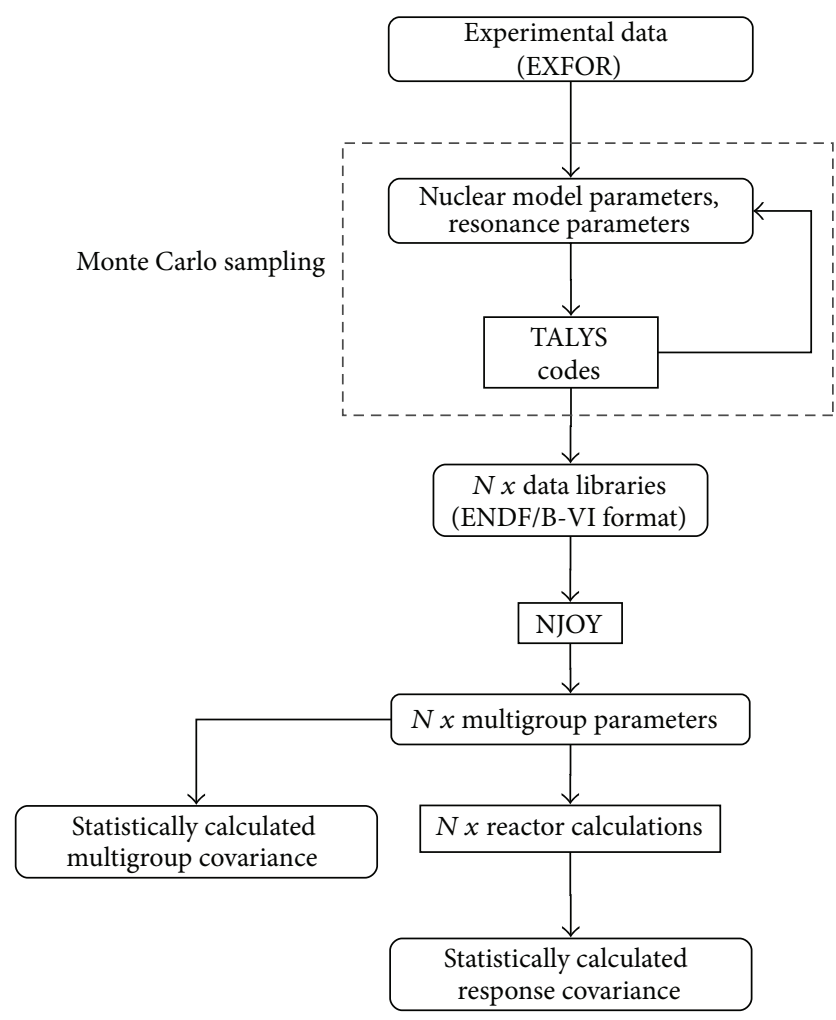

FIgURE 4: Simplified total Monte Carlo flowchart.

to redevelop such libraries from continuous nuclear data, the FCWG method provides a methodology for approximating the dilution impact on covariance.

\section{Dilution-Dependency of Uncertainty according to a Total Monte Carlo Technique}

Fundamentally, uncertainty associated with resonance crosssections results from uncertainty in resonance parameters $\left(E_{0}, \Gamma_{\gamma}\right.$, etc.). Variation of these parameters affects the crosssection in a more complex manner than the FCWG relationship assumed in Section 3. For example, uncertainty in nuclear data within a given energy group may affect not just the height of a peak, but also its position and relative width.

An alternative approach, which is addressed in this section, is to propagate uncertainties starting with the resonance parameters in an evaluated nuclear data library (ENDL) and observing the actual change in multigroup cross-section covariance at various dilutions using a cross-section processing code. A set of randomly generated TENDL $[16,17]$ files, produced using a total Monte Carlo (TMC) method [18, 19] (see Figure 4) in which uncertain resonance parameters are sampled by a Monte Carlo technique, are available through the TALYS project website. Whereas traditional multigroup lattice physics uncertainty techniques take multigroup crosssections as reference data, and multigroup cross-section covariance as uncertainty data, the TMC approach uses TENDL parameters as a reference and resonance parameter covariance as the input uncertainty data. Essentially, TMC initiates the uncertainty propagation procedure at a more fundamental level than multigroup methods, and consequently TMC can include in its propagation effects that are relevant prior to the calculation of multigroup parameters. We have used NJOY to produce multigroup cross-sections tables, indexed to dilution and temperature, for each of the random TENDL files for important reactions that may be influenced by dilution effects. For example, each TENDL file is processed with NJOY to arrive at a new WIMS-D4 multigroup library in 69 energy groups; each new WIMS library file consists of a unique set of cross-sections corresponding to the selected TENDL parental file and indexed to dilution. The 69-group structure of these libraries is identical to the 69-group WIMS structure used in Section 3. Given a large number of parental TENDL files ( $n$-files), the resultant WIMS-D4 files can then be statistically examined to ascertain the variance and covariance as a function of reaction and energy group. Since each output also includes tables of background cross-sections as a function of dilution, the covariance at each separate value of background crosssection can also be calculated. The TMC approach therefore facilitates the calculation of covariance of resonance group cross-sections at each value of background cross-section in the self-shielding table because the entire table and all of its entries are sampled $N$ times. The covariance of all table entries can be calculated according to

$$
\begin{aligned}
& \operatorname{cov}(a, b) \\
& =\frac{1}{N-1} \sum_{n=1}^{N}\left[\left(\sigma_{n}^{a}\left(\sigma_{0}\right)-\left\langle\sigma^{a}\left(\sigma_{0}\right)\right\rangle\right)\left(\sigma_{n}^{b}\left(\sigma_{0}\right)-\left\langle\sigma^{b}\left(\sigma_{0}\right)\right\rangle\right)\right] .
\end{aligned}
$$

By treating the uncertainty in this way the continuous energy cross-sections in the resonance range are subject to a broader scope of variation compared to the strictly up-and-down scaling of resonances assumed by the FCWG method. Using the TMC approach, therefore, the dilution-dependence of resonance group uncertainty can differ substantially compared to the approach used in Section 3. The relative standard deviation within the resonance groups at various dilutions compared to infinite dilution computed using TMC is shown in Figures 5 and 6 for $\sigma_{\gamma}^{\mathrm{U} 238}$ and $\sigma_{\gamma}^{\mathrm{U} 235}$, respectively. In contrast to the same data shown in Figures 2 and 3 in Section 3 which were derived through the FCWG assumption, the change in uncertainty with dilution using TMC exhibits significant differences. A striking disparity is seen in the dilution-dependency of $\sigma_{\gamma}^{\mathrm{U} 238}$ uncertainty, which increases at small background cross-sections, compared to the decrease in uncertainty that is predicted in Section 3. In general, the differences between the approaches increase as the dilution level decreases.

In addition to the uncertainty of each group, the change in covariance between resonance groups $a$ and $b$ can also be calculated from TMC statistically, again according to (10). The covariance of ${ }^{238} \mathrm{U}$ capture and elastic scatter crosssection resonance groups are shown in Figures 7, 8, 9, 10, 11, 12,13 , and 14 for four selected values of background crosssection. Likewise, the resonance group covariance of ${ }^{235} \mathrm{U}$ 


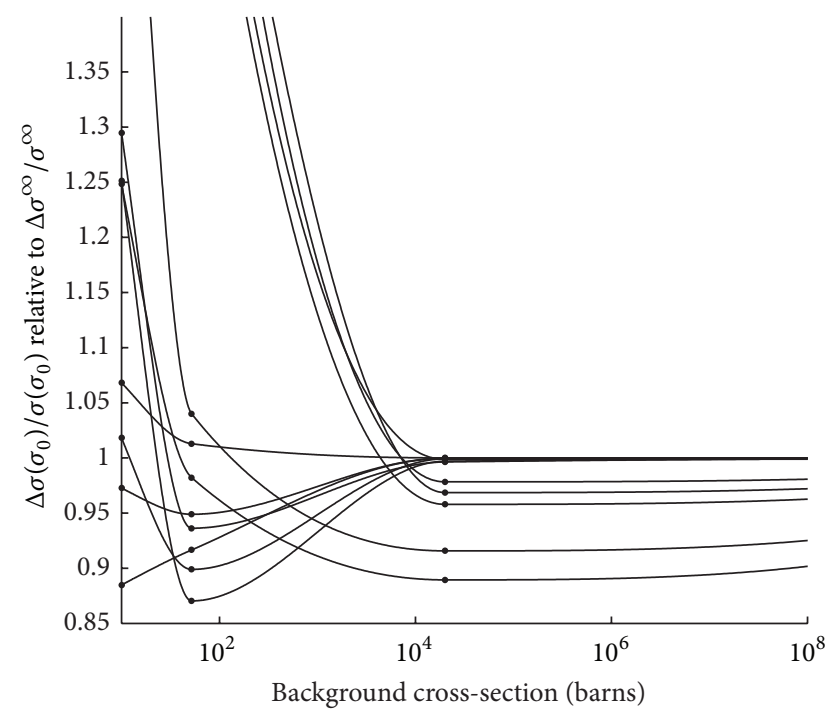

FIGURE 5: Resonance group $\Delta \sigma / \sigma$ versus dilution relative to infinite dilution of $\sigma_{\gamma}^{\mathrm{U} 238}$ using TMC.

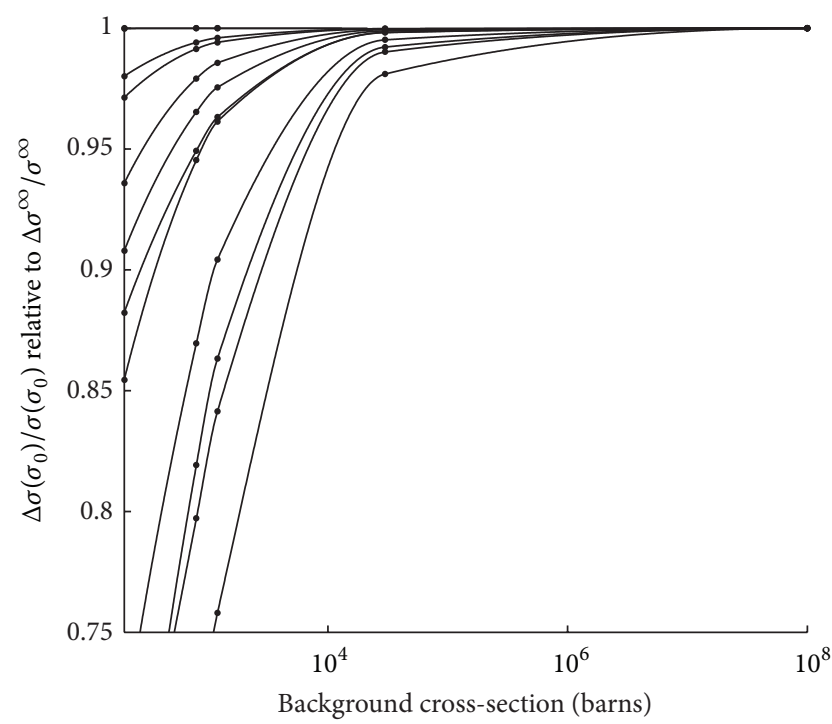

FIGURE 6: Resonance group $\Delta \sigma / \sigma$ versus dilution relative to infinite dilution of $\sigma_{\gamma}^{\mathrm{U} 235}$ using TMC.

capture and fission cross-sections are shown in Figures 15, 16, $17,18,19,20,21$, and 22 . For reference, the typical problem dilution of ${ }^{238} \mathrm{U}$ is approximately 50 barns for LWR and fast reactor systems [20] and the approximate dilution of ${ }^{235} \mathrm{U}$ is 800 barns. In the figures, the selected background cross-section values correspond to those used in tabulating the self-shielding tables in the WIMS-D4 multigroup library produced by the IAEA WLUP project.

While there is no substantial change in the shape of the covariance matrix for $\sigma_{\gamma}^{\mathrm{U} 235}$ and $\sigma_{f}^{\mathrm{U} 235}$, both $\sigma_{\gamma}^{\mathrm{U} 238}$ and $\sigma_{e}^{\mathrm{U} 238}$ show large changes in the off-diagonals of the matrix as dilution changes. Such effects cannot be adequately treated using a FCWG approximation. However, at this point in time the covariance contained within the TALYS data does not

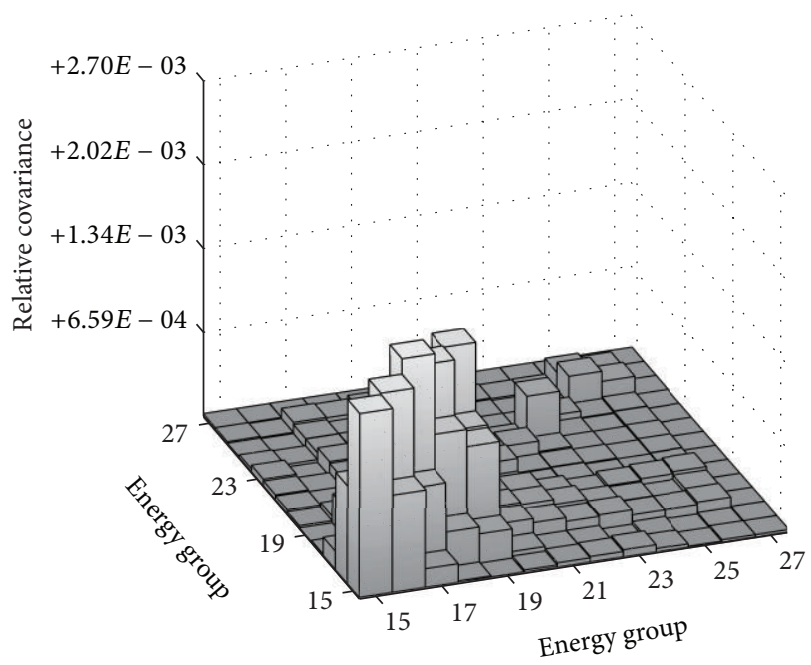

FIGURE 7: $\sigma_{\gamma}^{\mathrm{U} 238}$ covariance at infinite dilution.

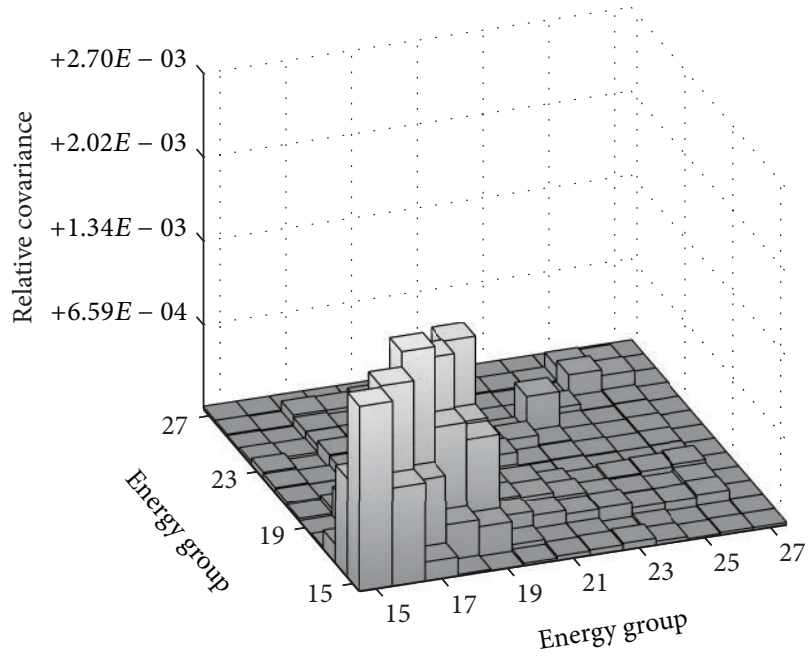

Figure 8: $\sigma_{\gamma}^{\mathrm{U} 238}$ covariance at $\sigma_{0}=2.0 E+4 b$.

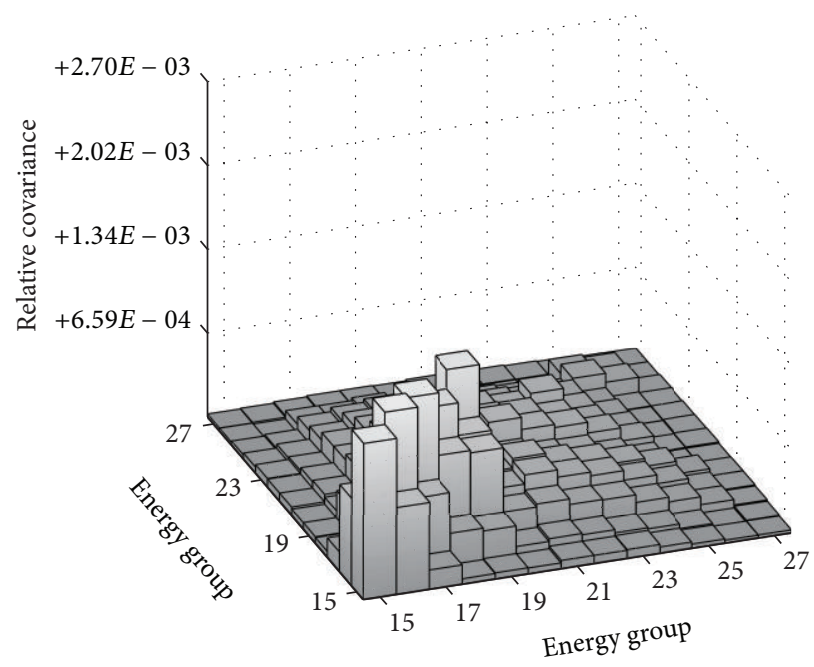

FIGURE 9: $\sigma_{\gamma}^{\mathrm{U} 238}$ covariance at $\sigma_{0}=52 b$. 


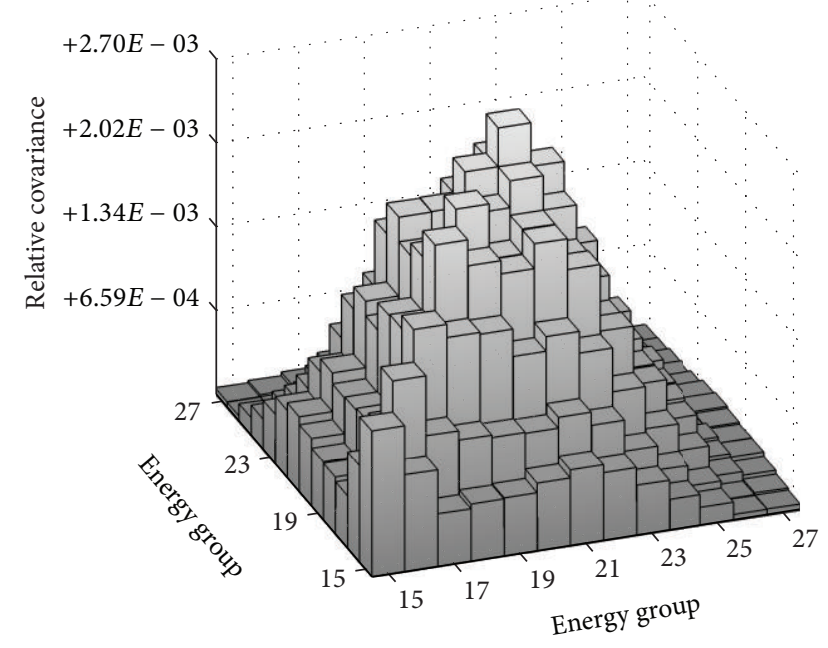

FIGURE 10: $\sigma_{\gamma}^{\mathrm{U} 238}$ covariance at $\sigma_{0}=10 b$.

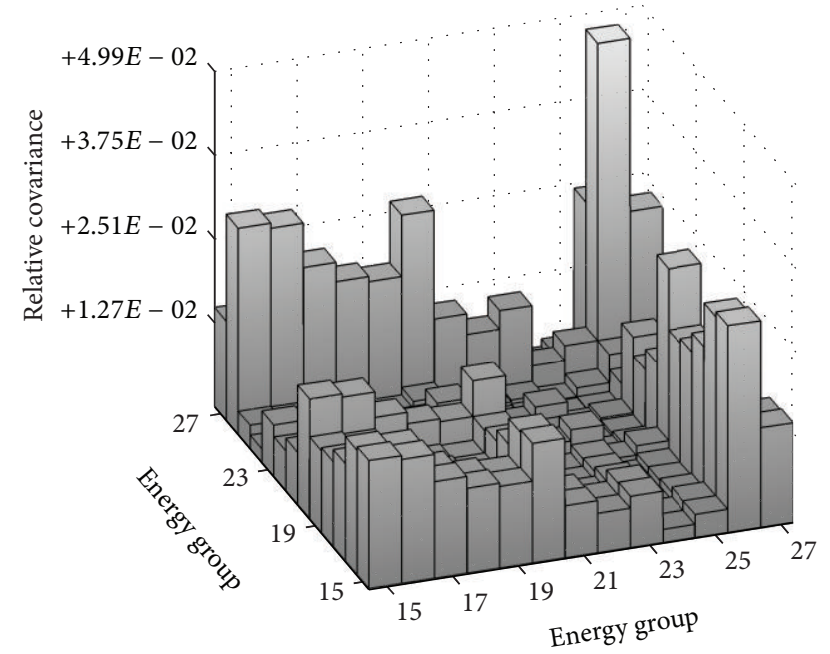

FIGURE 11: $\sigma_{e}^{\mathrm{U} 238}$ covariance at infinite dilution.

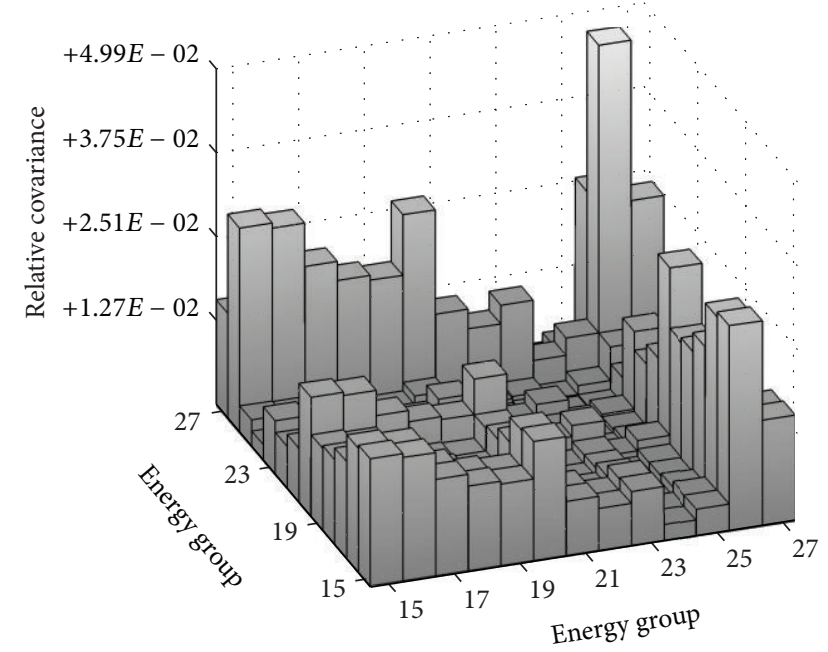

FIGURE 12: $\sigma_{e}^{\mathrm{U} 238}$ covariance at $\sigma_{0}=2.0 E+4 b$.

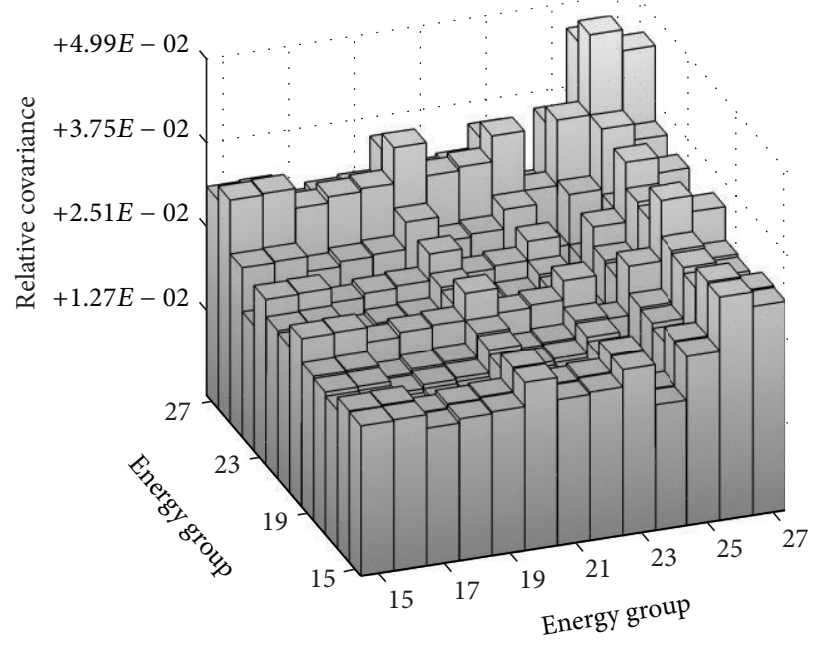

FIGURE 13: $\sigma_{e}^{\mathrm{U} 238}$ covariance at $\sigma_{0}=52 b$.

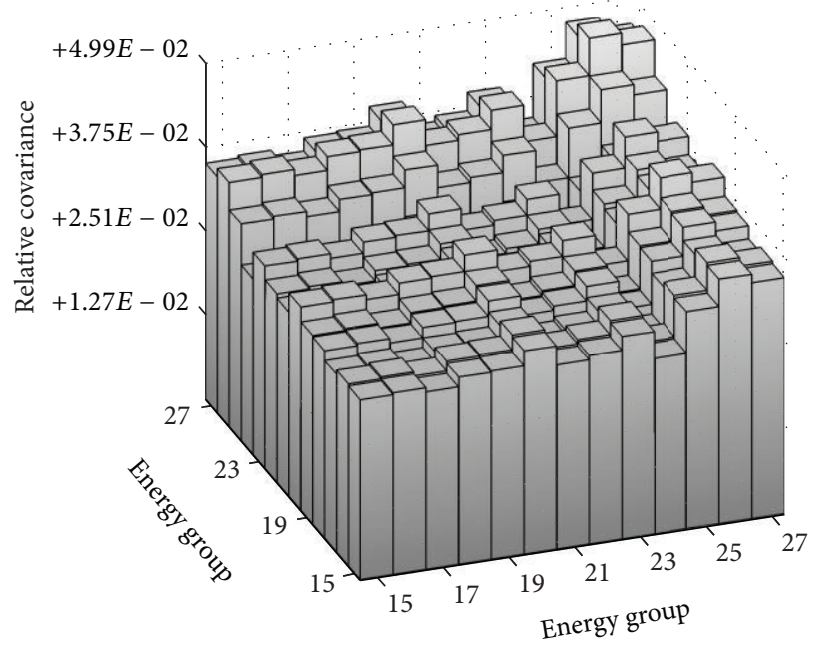

FIgURE 14: $\sigma_{e}^{\mathrm{U} 238}$ covariance at $\sigma_{0}=10 b$.

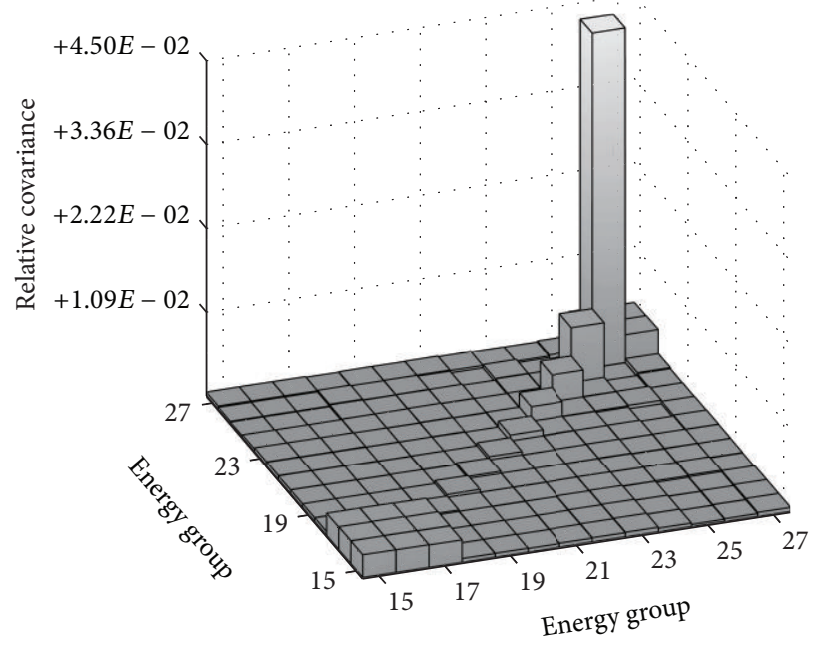

FIGURE 15: $\sigma_{\gamma}^{\mathrm{U} 235}$ covariance at infinite dilution. 


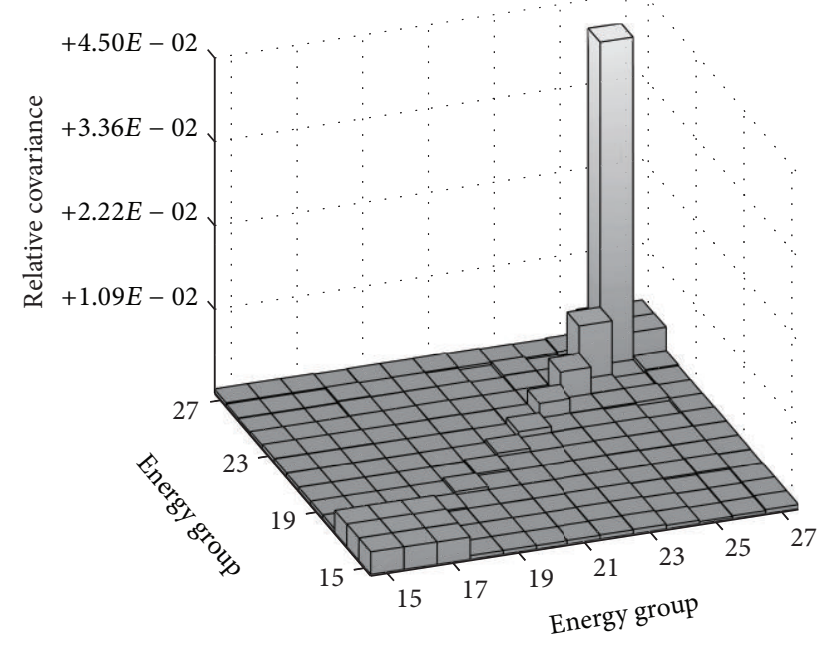

FIgURE 16: $\sigma_{\gamma}^{\mathrm{U} 235}$ covariance at $\sigma_{0}=3.0 E+4 b$.

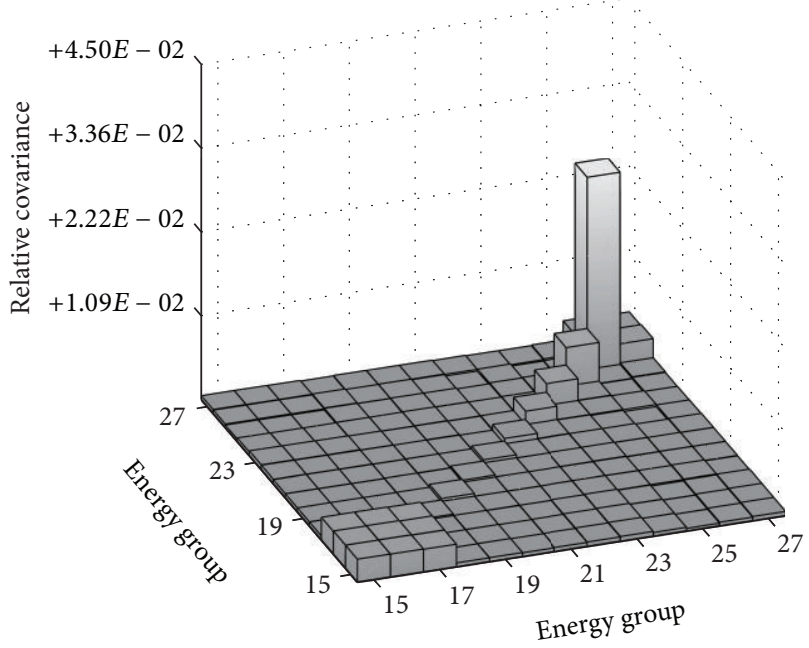

Figure 17: $\sigma_{\gamma}^{\mathrm{U} 235}$ covariance at $\sigma_{0}=800 b$.

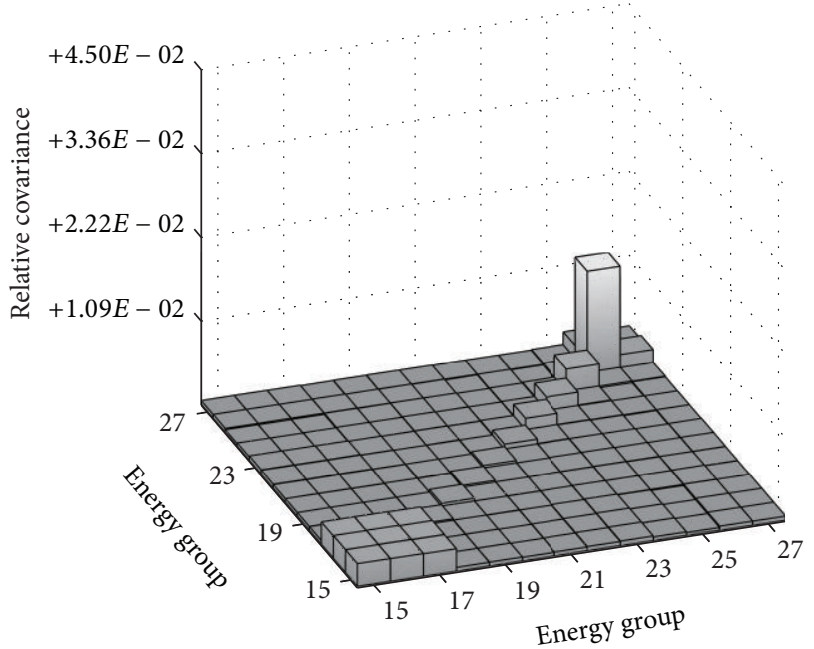

FIGURE 18: $\sigma_{\gamma}^{\mathrm{U} 235}$ covariance at $\sigma_{0}=200 b$.

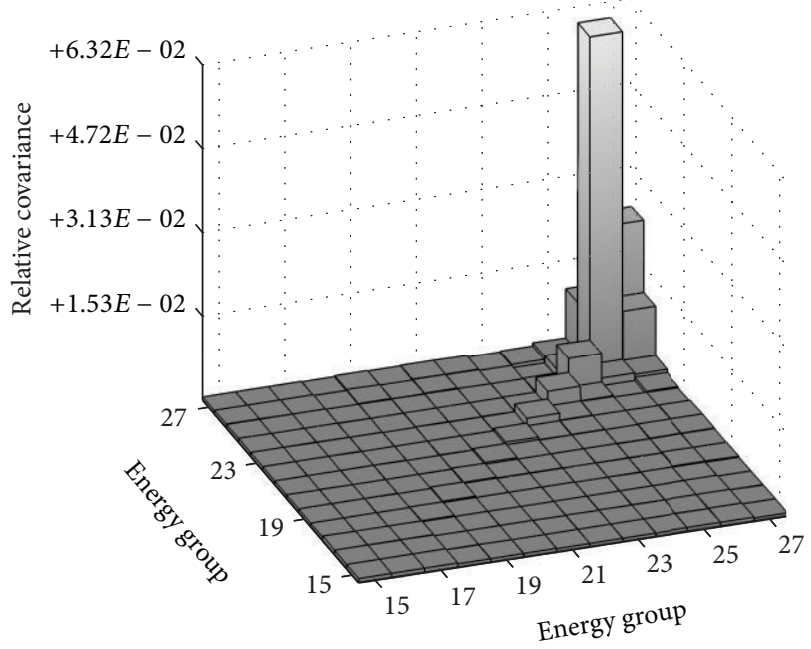

FIGURE 19: $\sigma_{f}^{\mathrm{U} 235}$ covariance at infinite dilution.

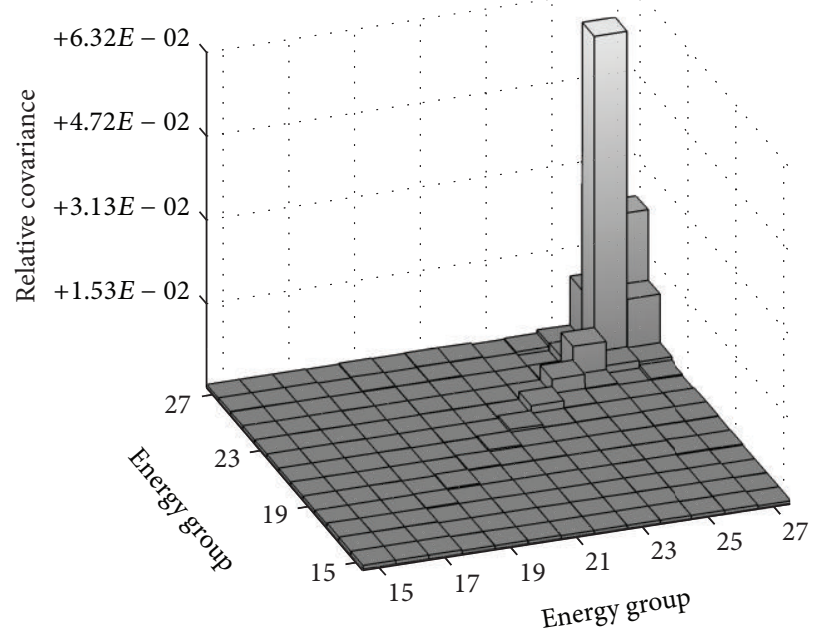

Figure 20: $\sigma_{f}^{\mathrm{U} 235}$ covariance at $\sigma_{0}=3.0 E+4 b$.

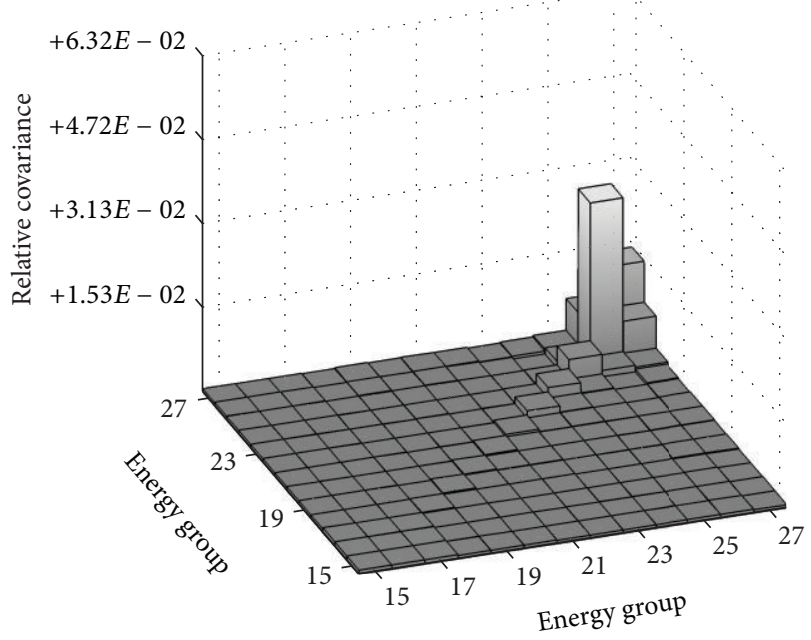

FIGURE 21: $\sigma_{f}^{\mathrm{U} 235}$ covariance at $\sigma_{0}=800 b$. 


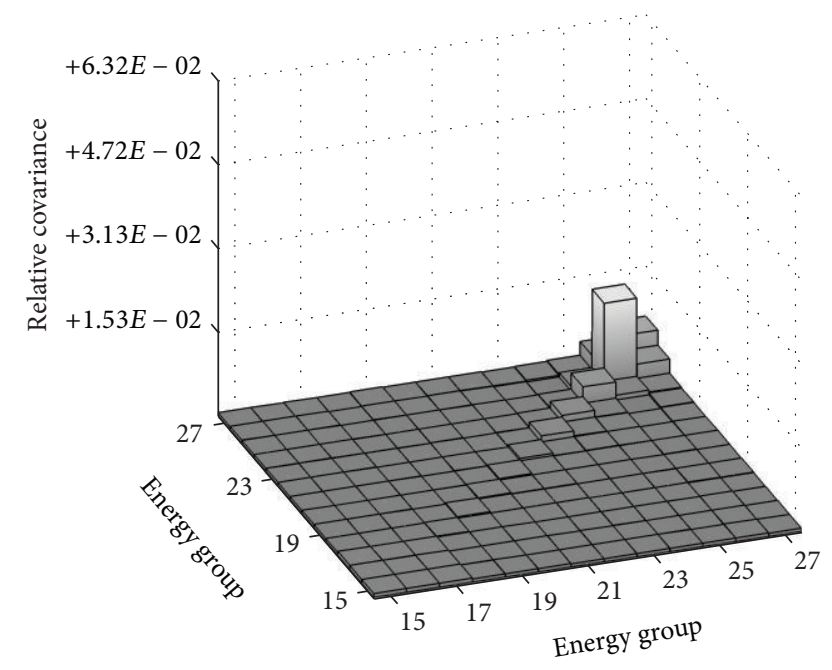

Figure 22: $\sigma_{f}^{\mathrm{U} 235}$ covariance at $\sigma_{0}=200 b$.

contain all possible fission products and actinides, and therefore its applications to reactor-core analysis and uncertainties including burnup are limited. Therefore while deviations exist between the methods, other practical limitations may dictate the approach used. The above demonstrates an important subtlety that the dilution effects the covariance itself, in addition to the well-known effects that it has on the reference cross-sections, through the self-shielding process. Therefore, in a complete treatment of uncertainty using multigroup methods it may be necessary to have covariance data indexed with dilution.

\section{Impact on OECD/NEA UAM Benchmark Test Case}

Sections 3 and 4 have described two ways to evaluate multigroup covariance at a problem dilution. The method in Section 3, using the FCWG assumption, can be used to calculate a correction to an infinite-dilution covariance and can be implemented in a lattice calculation when only multigroup data are available, for example, when legacy issues surrounding a data set make it difficult to reconstruct based only on continuous spectra or where multigroup crosssections have undergone rigorous parameter adjustment based on experimental data. It is therefore straightforward to implement in many lattice-type codes whose inputs are multigroup in nature, for example, in DRAGON [10] and other code packages [21]. The method described in Section 4 involved a TMC approach where covariance at problem dilution is computed by Monte Carlo sampling of evaluated nuclear data and processing the samples using a cross-section code such as NJOY.

In this section, both approaches are used to propagate cross-section uncertainties through a boiling water reactor (BWR) fuel cell, specified in Table 2. The cell specifications are given as part of the uncertainty analysis in modelling (UAM) benchmark sponsored by the OECD/NEA. The first exercises of the benchmark involve the assessment of uncertainties for a variety of fuel cell configurations and energy
TABLE 2: BWR lattice specifications.

\begin{tabular}{lc}
\hline Parameter & Value \\
\hline Unit cell pitch $[\mathrm{mm}]$ & 18.750 \\
Fuel pellet diameter $[\mathrm{mm}]$ & 12.1158 \\
Fuel pellet material & $\mathrm{UO}_{2}$ \\
Fuel density $\left[\mathrm{g} / \mathrm{cm}^{3}\right]$ & 10.420 \\
Fuel enrichment $[\mathrm{w} / \mathrm{o}]$ & 2.93 \\
Cladding outside diameter $[\mathrm{mm}]$ & 14.3002 \\
Cladding thickness $[\mathrm{mm}]$ & 0.9398 \\
Cladding material & Zircaloy-2 \\
Cladding density $\left[\mathrm{g} / \mathrm{cm}^{3}\right]$ & 6.55 \\
Gap material & $\mathrm{He}$ \\
Moderator material & $\mathrm{H}_{2} \mathrm{O}$ \\
\hline Reactor conditions & Value \\
\hline Fuel temperature $[\mathrm{K}]$ & 900.0 \\
Cladding temperature $[\mathrm{K}]$ & 600.0 \\
Moderator temperature $[\mathrm{K}]$ & 557.0 \\
Moderator density $\left[\mathrm{kg} / \mathrm{m}^{3}\right]$ & 460.72 \\
\hline
\end{tabular}

group structures. As point of reference, the uncertainty propagation was also conducted using infinite-dilution covariance directly, with no regard to its dilution-dependency.

Uncertainties were propagated through the BWR fuel cell lattice specified in Table 2 using sensitivity and uncertainty tool [10] and the lattice code DRAGON [22]. Explicit, indirect, and background cross-section mechanisms (refer to Section 1) were all treated in the analysis. The dilutiondependency mechanism of the cross-section covariance was computed using FCWG or TMC. Additional comparisons are also performed where covariance is applied without any selfshielding effects. In all cases, the multigroup covariance used originated from the TENDL libraries. In the infinite-dilution case, where no dilution-dependency of covariance was considered, an infinite-dilution covariance was calculated from the TENDL libraries and applied directly to the post selfshielded cross-sections used by DRAGON. In the FCWG case, the same infinite-dilution covariance was corrected to its problem dilution according to (8) and (9) and as described in [22]. For the TMC case, the problem-dependent covariance was calculated from the output of the NJOY flux calculator applied to the TENDL libraries.

Table 3 shows the relative uncertainty of $k_{\infty}(\Delta k / k)$ resulting from the uncertainty of the resonance groups of selected cross-sections when their dilution-dependency was either not considered (infinite-dilution covariance) or considered using the FCWG or TMC methods. The table shows substantial difference between the resonance group covariance contributions to $\Delta k / k$ for the selected cross-sections for the two covariance propagation methods. The data also suggest that the FCWG method provides only a small change in $k_{\infty}$ uncertainty compared to neglecting the dilution-dependency of the covariance completely. To some extent the small differences between infinite dilution and FCWG may explain why historically some uncertainty propagation methods did not process the covariance library through the self-shielding 
TABLE 3: $k_{\infty}$ uncertainty attributable to resonance group covariance of selected cross-sections.

\begin{tabular}{lccc}
\hline \multirow{2}{*}{$\begin{array}{l}\text { Uncertain } \\
\text { parameter }\end{array}$} & \multirow{2}{*}{ Explicit/infinite dilution } & \multicolumn{2}{c}{$\Delta k / k$} \\
\hline$\sigma_{\gamma}^{\mathrm{U} 238}$ & $0.162 \%$ & FCWG & TMC \\
$\sigma_{\gamma}^{\mathrm{U} 235}$ & $0.202 \%$ & $0.156 \%$ & $0.302 \%$ \\
$\sigma_{f}^{\mathrm{U} 235}$ & $0.201 \%$ & $0.196 \%$ & $0.141 \%$ \\
\hline
\end{tabular}

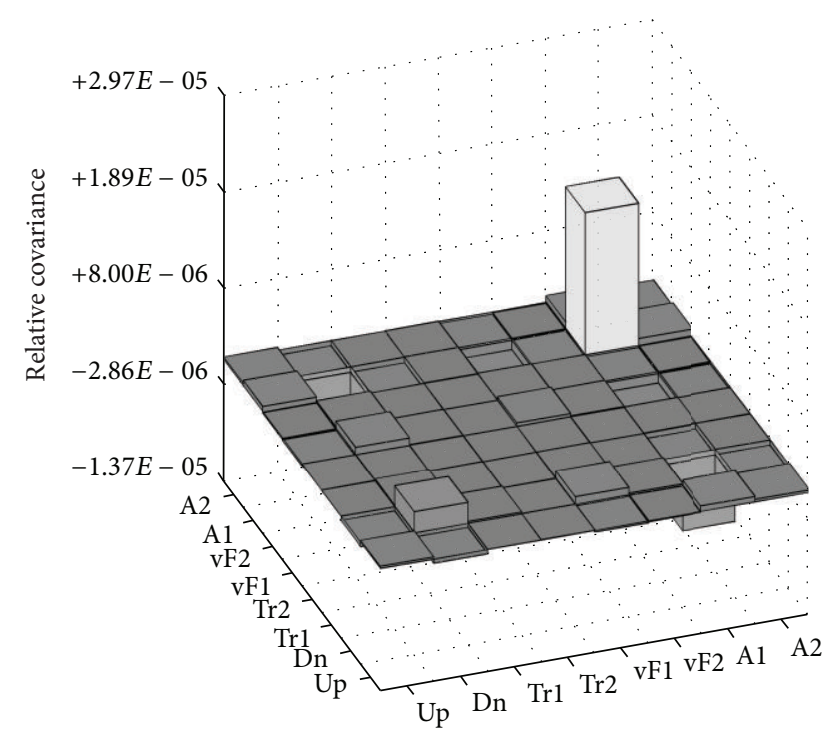

FIGURE 23: Homogenized property covariance due to infinitedilution $\sigma_{\gamma}^{\mathrm{U} 238}$ covariance.

calculation but rather chose to apply it after self-shielding of the reference cross-sections.

A comparison of the uncertainty associated with twogroup, homogenized properties shows similar characteristics to that of $k_{\infty}$. Figures 23, 24, and 25 show the covariance of homogenized, two-group cell properties due to the propagation of the covariance of all energy groups of $\sigma_{\gamma}^{\mathrm{U} 238}$, while neglecting dilution-dependency of its covariance, and when using the FCWG and TMC methods. Likewise, Figures 26, 27, and 28 show the same of $\sigma_{\gamma}^{\mathrm{U} 235}$ and Figures 29,30 , and 31 show that of $\sigma_{f}^{\mathrm{U} 235}$. In the figures, "Up," "Dn," “ $\operatorname{Tr} 1$," “Tr2," "vF1," "vF2," "A1," and "A2" refer to up-scatter, down-scatter, transport cross-section (groups 1 and 2), fission-yield cross-section (groups 1 and 2), and absorption cross-section (groups 1 and 2), respectively. As with the $k_{\infty}$ uncertainty, the FCWG method offers minimal difference to the results when the dilution-dependency of the input covariance was neglected. The TMC method, however, illustrates a significant change in lattice calculation output covariance versus dilution.

\section{Conclusions}

It has been shown that the uncertainty in multigroup crosssections varies according to its problem-dependent background cross-section and in fact covariance information in

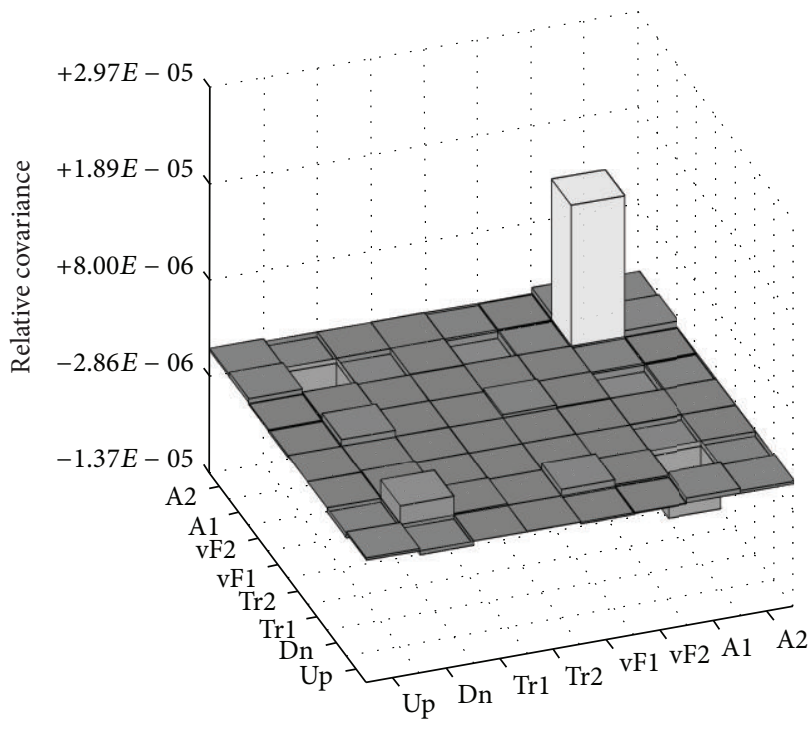

FIGURE 24: Homogenized property covariance due to $\sigma_{\gamma}^{\mathrm{U} 238}$ covariance using FCWG.

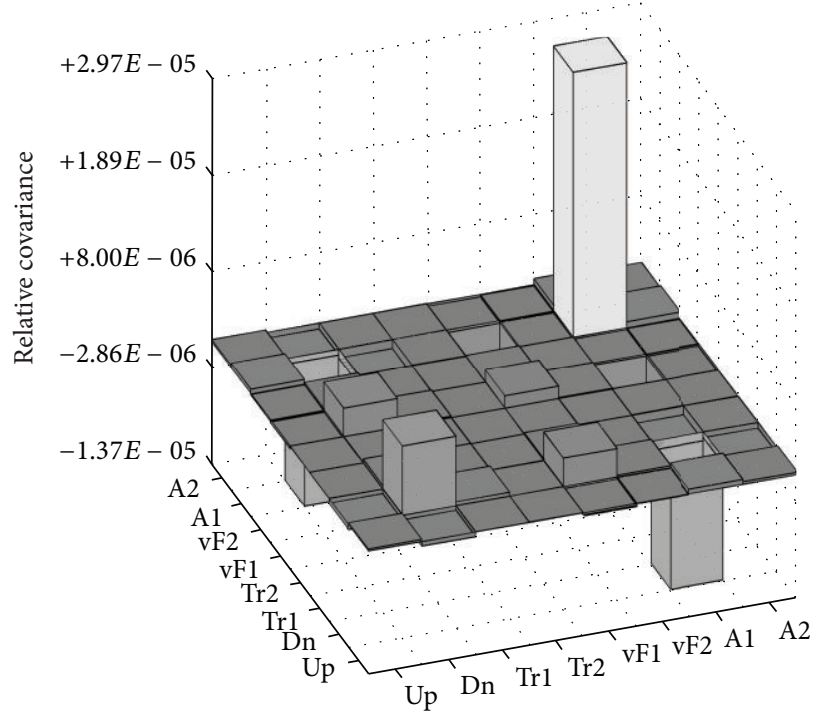

FIGURE 25: Homogenized property covariance due to $\sigma_{\gamma}^{\mathrm{U} 238}$ covariance using TMC.

multigroup format should have some index to dilution. Two methods to account for this dilution-dependency include a total Monte Carlo approach and an approach which is straightforward to implement using only multigroup data according to a fully correlated within-group assumption of the underlying data.

By propagating sample TENDL files through NJOY, the covariance of multigroup cross-sections could be computed at various background cross-sections. Then using this information, the exact covariance at a problem-specific dilution can be included. Using total Monte Carlo, the covariance matrix for resonance groups of several selected reactions showed potentially significant differences compared to 


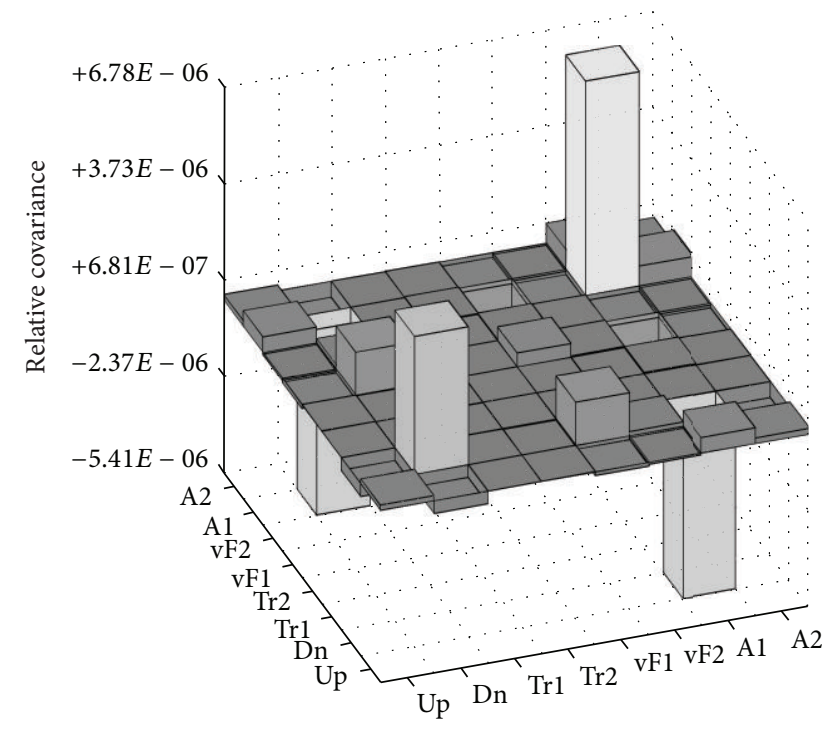

FIGURE 26: Homogenized property covariance due to infinitedilution $\sigma_{\gamma}^{\mathrm{U} 235}$ covariance.

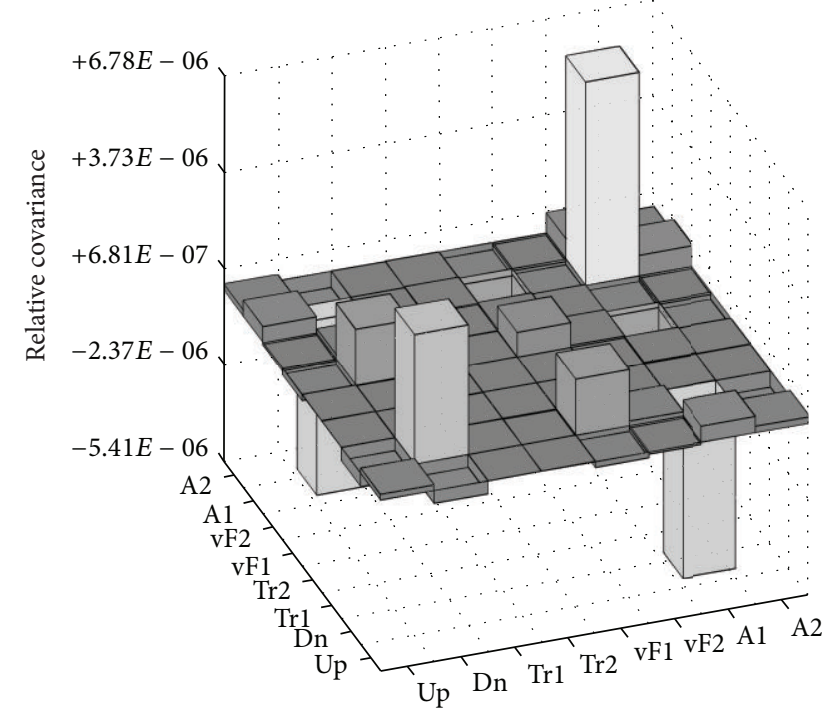

FIgURE 27: Homogenized property covariance due to $\sigma_{\gamma}^{\mathrm{U} 235}$ covariance using FCWG.

infinite-dilution uncertainty assumptions when the problem dilution decreased.

For a BWR fuel cell lattice test case, the simpler method for treating the covariance dilution-dependency, using the fully correlated within-group assumption, did not agree with the results obtained using the more rigorous total Monte Carlo approach. In addition, cases where no self-shielding effects are included also did not agree with the TMC method. Using total Monte Carlo, there was a nontrivial change in the uncertainty contributed by selected reactions to that of $k_{\infty}$, when compared to the same calculations when the infinite-dilution covariance was used. Consequently, infinitedilution covariance matrices may not be suitable proxies for

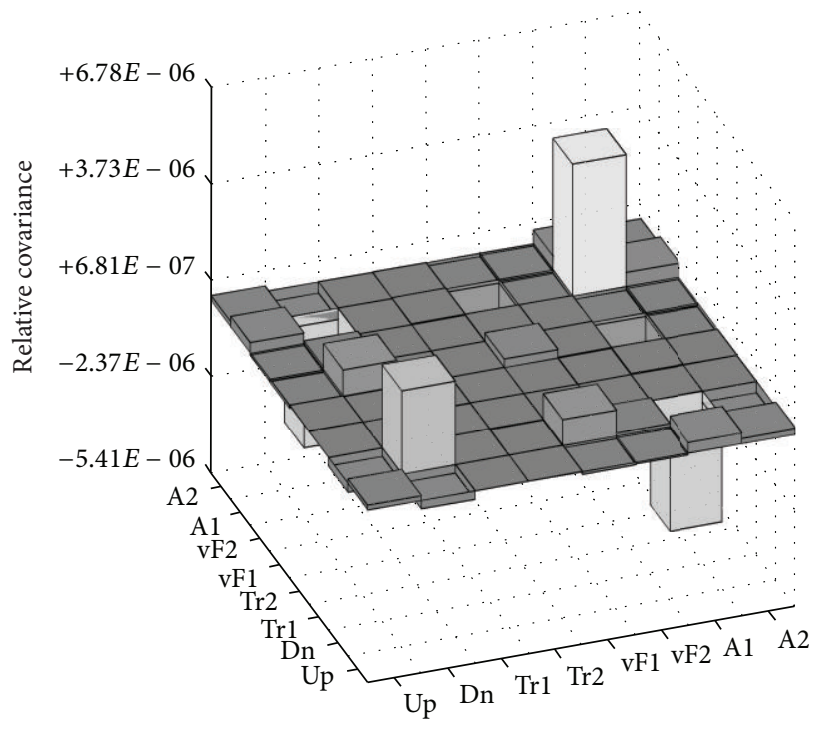

FIGURE 28: Homogenized property covariance due to $\sigma_{\gamma}^{\mathrm{U} 235}$ covariance using TMC.

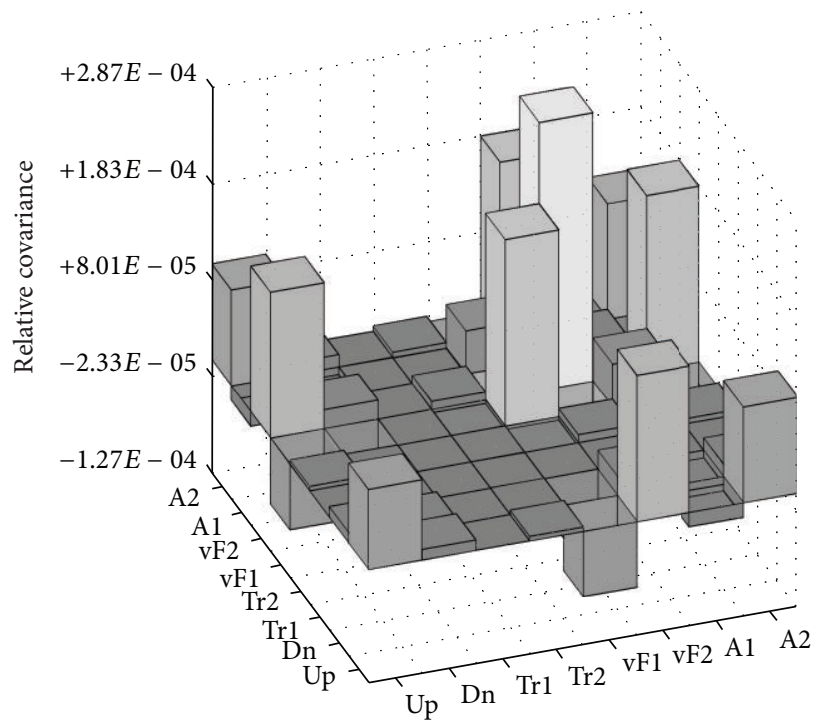

Figure 29: Homogenized property covariance due to infinitedilution $\sigma_{f}^{\mathrm{U} 235}$ covariance.

the covariance of multigroup cross-sections at lower dilutions, though further investigation is necessary to evaluate their impact on full-core calculations. Of particular interest may be cross-sections of ${ }^{238} \mathrm{U}$, which contain significant uncertainties and also belong to a nuclide that appears in thermal reactor fuel mixtures at especially low dilution. Given the relatively large changes in the uncertainties that occur as a result of dilution and the need to perform such calculations for a range of fission products during a burn-up cycle (and even further if thermal hydraulic branch points are considered) the use of extended nuclear data libraries such as TALYS will become increasingly more important. Continued development of TALYS-type information (with 


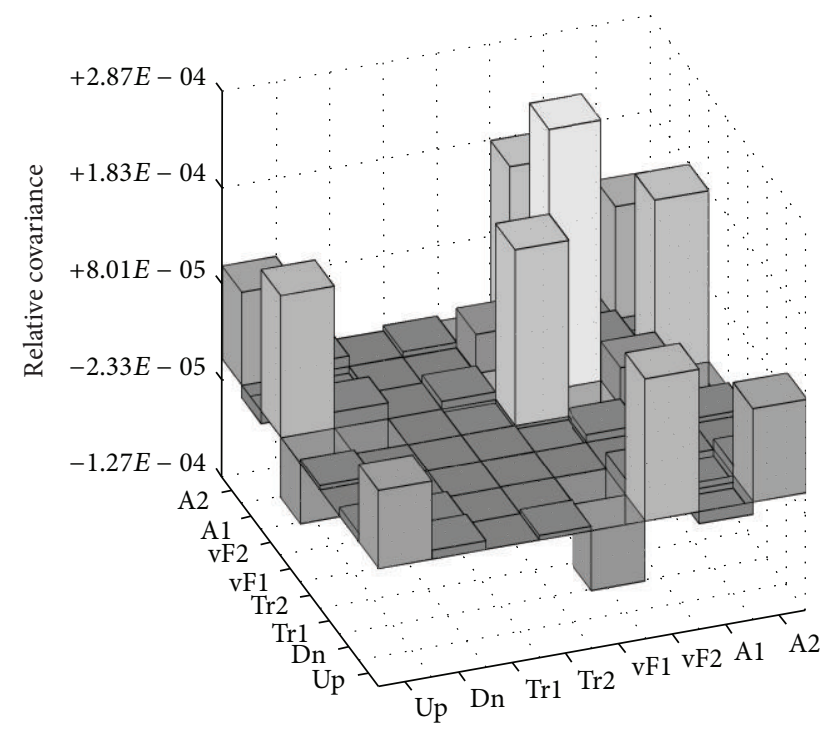

FIgURE 30: Homogenized property covariance due to $\sigma_{f}^{\mathrm{U} 235}$ covariance using FCWG.

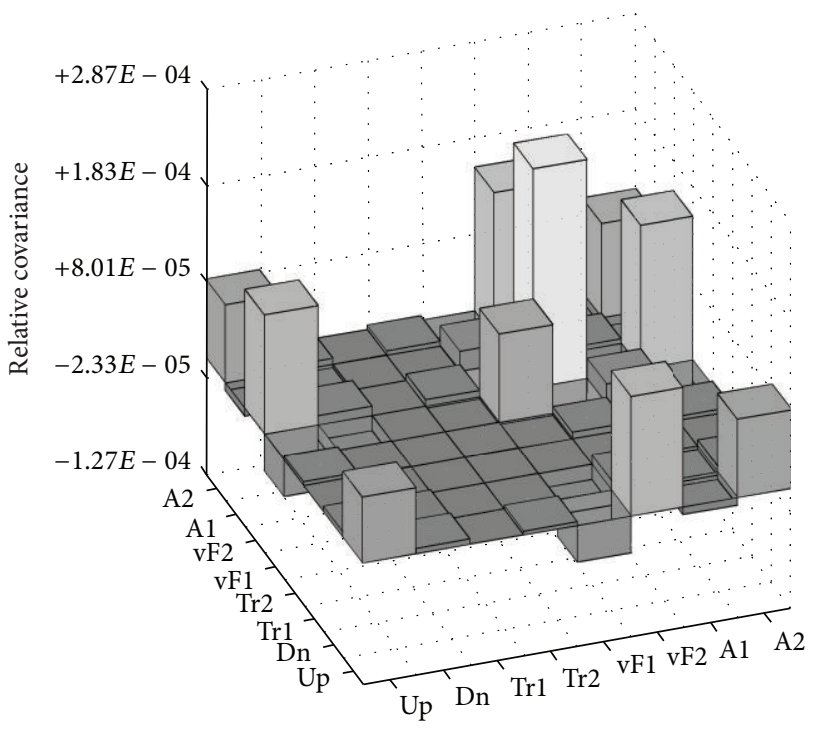

FIGURE 31: Homogenized property covariance due to $\sigma_{f}^{\mathrm{U} 235}$ covariance using TMC.

expanded ranges of isotopes and temperatures) is therefore recommended. Since one of the goals of the UAM project is to apply uncertainty methods to existing reactor calculations such developments will be critical to establishing the accuracy of lower order methods such as FCWG or for continued total Monte Carlo applications. Finally, the approximate methods pursued in this paper, namely,

(i) fully correlated within group where the effect of selfshielding is included in the reference cross-sections and the effects of uncertainty in resonances are included through a perturbed background crosssection, (ii) applications where self-shielding is performed on the reference cross-sections but the covariance library is left unperturbed,

should be applied with caution since they appear to deviate from the TMC approach for strong absorbers at low dilution. However the advantage of such approximate methods is that they can be applied easily within existing reactor analysis tools and can be applied to preexisting multigroup libraries for a range of burnups and branch points. Therefore, the direct application of uncertainties to full-scale analyses still remains an active topic of research within the OECD-NEA UAM project.

\section{Conflict of Interests}

The authors declare that there is no conflict of interests regarding the publication of this paper.

\section{References}

[1] K. Ivanov, M. Avramova, S. Kamerow et al., Benchmark for Uncertainty Analysis in Modeling (UAM) for Design, Operation and Safety Analysis of LWRs, vol. 1, Version 2.0, OECD Nuclear Energy Agency, 2011.

[2] E. Greenspan, "Sensitivity functions for uncertainty analysis," in Advances in Nuclear Science and Technology, J. Lewins and M. Becker, Eds., vol. 14, pp. 193-246, Plenum Press, New York, NY, USA, 1982.

[3] E. Greenspan, "New developments in sensitivity theory," in Advances in Nuclear Science and Technology, J. Lewins and M. Becker, Eds., vol. 14, pp. 313-361, Plenum Press, New York, NY, USA, 1982.

[4] E. Greenspan and Y. Karni, "Spectral fine-structure effects on material and doppler reactivity worths," Nuclear Science and Engineering, vol. 69, no. 2, pp. 169-190, 1979.

[5] B. T. Rearden, TSUNAMI-1D: Control Module for One-Dimensional Cross-Section Sensitivity and Uncertainty Analysis for Criticality, vol. 1, ORNL/TM-2005/39, Version 6, Section C8, 2009.

[6] M. L. Williams and B. T. Rearden, "SCALE-6 sensitivity/uncertainty methods and covariance data," Nuclear Data Sheets, vol. 109, no. 12, pp. 2796-2800, 2008.

[7] B. T. Rearden, M. L. Williams, M. A. Jessee, D. E. Mueller, and D. A. Wiarda, "Sensitivity and uncertainty analysis capabilities and data in SCALE," Nuclear Technology, vol. 174, no. 2, pp. 236-288, 2011.

[8] M. L. Williams, D. Wiarda, G. Arbanas, and B. L. Broadhead, Scale Nuclear Data Covariance Library ORNL/TM-2005/39, Version 6, vol. 3, section M19, Oak Ridge National Laboratory, 2009.

[9] R. E. MacFarlane and A. C. Kahler, "Methods for processing ENDF/B-VII with NJOY," Nuclear Data Sheets, vol. 111, no. 12, pp. 2739-2890, 2010.

[10] M. R. Ball, D. R. Novog, and J. C. Luxat, "Analysis of implicit and explicit lattice sensitivities using DRAGON," Nuclear Engineering and Design, vol. 265, pp. 1-12, 2013.

[11] R. E. MacFarlane, "Neutron slowing down and thermalization," in Handbook of Nuclear Engineering, D. Cacuci, Ed., pp. 189278, 2010. 
[12] I. I. Bondarenko, Group Constants for Nuclear Reactor Calculations, Consultants Bureau, New York, NY, USA, 1964.

[13] A. Trkov, G. Zerovnik, L. Snoj, and M. Ravnik, "On the selfshielding factors in neutron activation analysis," Nuclear Instruments and Methods in Physics Research A, vol. 610, no. 2, pp. 553-565, 2009.

[14] C. J. Taubman, “The WIMS 69-Group Library Tape 166259," Tech. Rep. AEEW-M1324, U.K. Atomic Energy Authority, Winfrith, Newburgh, UK, 1975.

[15] F. Leszczynksi, D. López Aldama, and A. Trkov, WIMS-D Library Update: Final Report of a Coordinated Research Project, International Atomic Energy Agency, Vienna, Austria, 2007.

[16] D. Rochman, S. van der Marck, J. Kopecky et al., “TENDL-2012: TALYS-based evaluated nuclear data library," http://www.talys .eu/tendl-2012/.

[17] A. J. Koning and D. Rochman, "Modern nuclear data evaluation with the TALYS code system," Nuclear Data Sheets, vol. 113, no. 12, pp. 2927-2934, 2012.

[18] D. Rochman and A. J. Koning, "Propagation of ${ }^{235,236,238}$ Uand ${ }^{239}$ pu nuclear data uncertainties for a typical pwr fuel element," Nuclear Technology, vol. 179, no. 3, 2012.

[19] D. Rochman, A. J. Koning, S. C. van der Marck, A. Hogenbirk, and C. M. Sciolla, "Nuclear data uncertainty propagation: perturbation vs. Monte Carlo," Annals of Nuclear Energy, vol. 38, no. 5, pp. 942-952, 2011.

[20] J. Barre and A. Khairallah, "Importance of resonance parameters of fertile nuclei and of $239 \mathrm{Pu}$ isotope for fast power reactors," in Proceedings of the Specialist Meeting on Resonance Parameters of Fertile Nuclei and 239 Pu Isotope, P. Ribon, Ed., pp. 19-42, Saclay, France, May 1974.

[21] S. M. Bowman, "Overview of the SCALE code system," Transactions of the American Nuclear Society, vol. 97, pp. 589-591, 2007.

[22] G. Marleau, A. Hébert, and R. Roy, "A user guide for DRAGON 3.06," Tech. Rep. IGE-174 Rev. 1, École Polytechnique de Montréal, 2012. 


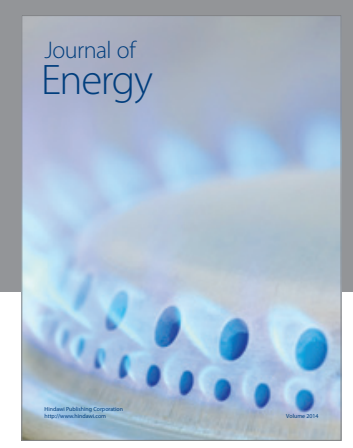

Journal of

Industrial Engineering
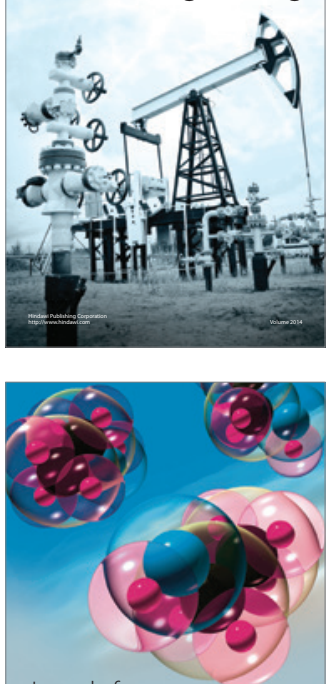

Fuels
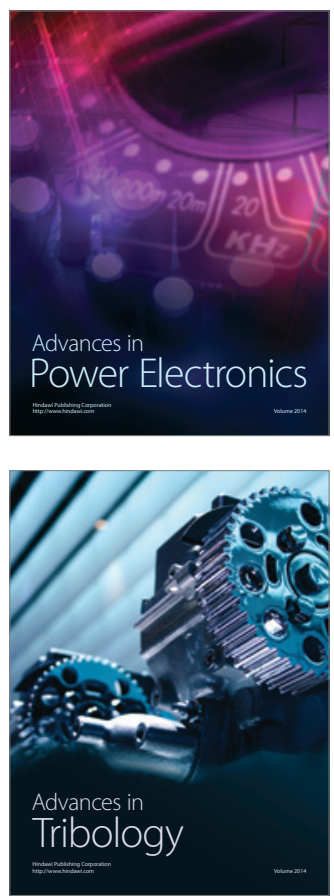

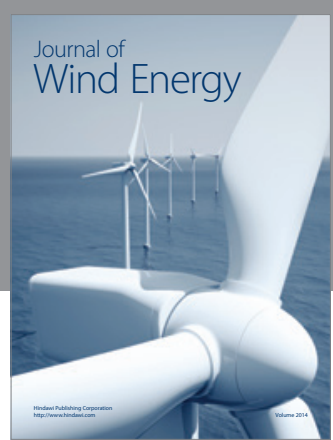

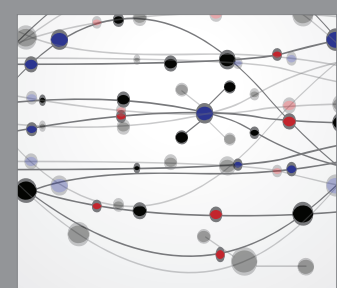

The Scientific World Journal

Submit your manuscripts at http://www.hindawi.com

Journal of

Structures
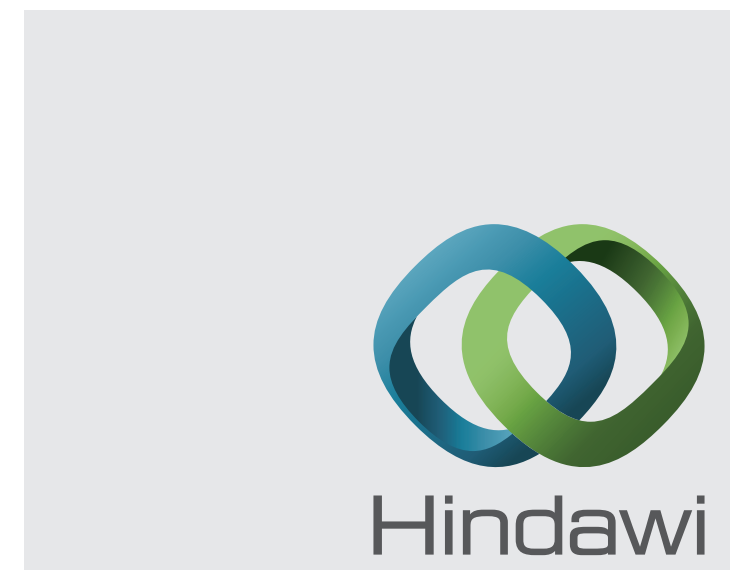

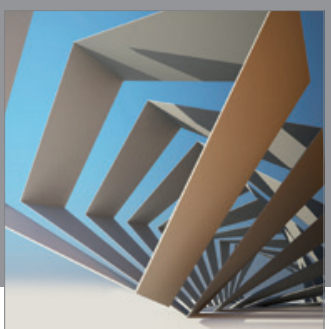

Rotating

Machinery
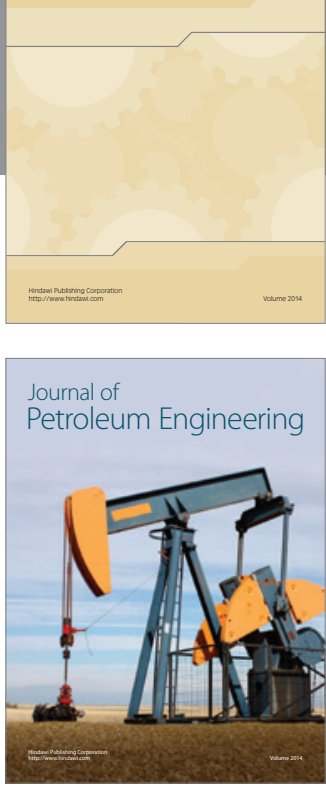

Journal of

Solar Energy
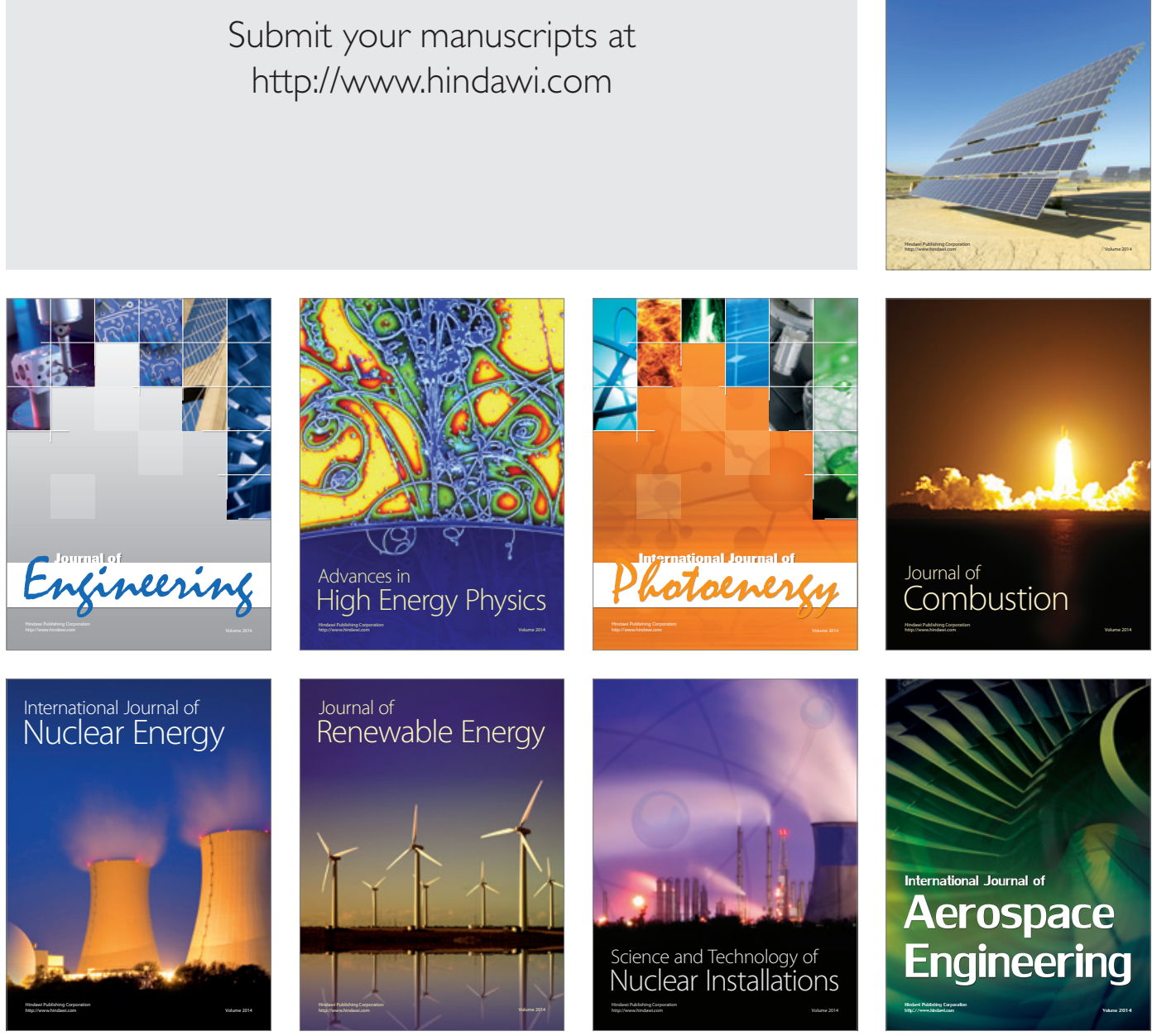